\title{
Implicit and explicit integration schemes in the anisotropic bounding surface plasticity model for cyclic behaviours of saturated clay
}

\author{
Cun $\mathrm{Hu}^{\mathrm{a}}{ }^{\mathrm{b}}$, Haixiao $\mathrm{Liu}^{\mathrm{a}, *}$ \\ a School of Civil Engineering, Tianjin University, Tianjin 300072, China \\ b Institute of Mechanics, Chinese Academy of Sciences, Beijing 100190, China
}

\section{A R T I C L E I N F O}

\section{Article history:}

Received 30 March 2013

Received in revised form 26 July 2013

Accepted 26 July 2013

Available online 23 August 2013

\section{Keywords:}

Bounding surface plasticity model

Implicit integration

Explicit integration

Cyclic behaviour

Saturated clay

\begin{abstract}
A B S T R A C T
Two integration algorithms, namely the implicit return mapping and explicit sub-stepping schemes, are adopted in the anisotropic bounding surface plasticity model for cyclic behaviours of saturated clay and are implemented into finite element code. The model is a representative of a series of bounding surface models that have typical characteristics, including isotropic and kinematic hardening rules and a rotational bounding surface to capture complex but important cyclic behaviours of soils, such as cyclic shakedown and degradation. However, there is no explicit current yield surface in the model to which the conventional implicit algorithm returns the stress state back or the sub-stepping integration corrects the drift of the stress state. Hence, necessary modifications have been made for both of the integration schemes. First, the image stress point is mapped or corrected to the bounding surface instead of mapping back or correcting the stress state to the yield surface. Second, the unloading-loading criterion is checked to determine the image stress point rather than checking the yield criterion after giving the trial stress state in a conventional way. Comparative studies on the accuracy, stability and efficiency of the two integration schemes are conducted not only at the element level but also in solving boundary value problems of monotonic and cyclic bearing behaviours of rigid footings on saturated clay. For smaller strain increments, there is no significant difference in the accuracy between the two integration schemes, but the explicit integration shows a higher efficiency and accuracy. For relatively larger increments, the implicit return mapping algorithm presents good accuracy and more robustness, while the sub-stepping algorithm shows deteriorating accuracy and suffers the convergence problem. With the tolerance used in the present model, the bearing capacity of the rigid footing predicted by the return mapping algorithm is closer to the available analytical and numerical solutions, while the bearing capacity predicted by the sub-stepping algorithm shows a marginal increase.
\end{abstract}

(c) 2013 Elsevier Ltd. All rights reserved.

\section{Introduction}

The response simulation of offshore structures embedded in seabed soils under cyclic loading still faces significant obstacles. First, it requires efficient and accurate constitutive models that reflect important cyclic behaviours of seabed soils, such as the hysteretic property, initial anisotropy, cyclic shakedown and stiffness degradation as well as the accompanying accumulation of plastic strain and pore pressure [1-3]. However, to capture all of these important but complex behaviours makes the constitutive model more lengthy and complicated. Moreover, in order to be applicable to offshore geotechnical calculations, the constitutive model requires efficient and robust numerical implementations, whereas the integration scheme of the incremental constitutive relations is

\footnotetext{
* Corresponding author. Tel.: +86 2227401510.

E-mail address: liuhx@tju.edu.cn (H. Liu).
}

the cornerstone that controls the accuracy, stability and efficiency of the calculations.

Existing approaches for stress integration of elasto-plastic constitutive models are generally classified as implicit and explicit schemes. Implicit algorithms that are based on the closest point projection or the return mapping [4-10] require a consistent tangent operator that corresponds to the final stress state of the integration increment. This arrangement means that an iterative calculation of the final stress state is needed. Explicit algorithms such as the algorithm with automatic error control and substepping [11-14] require a continuum tangent operator that corresponds only to the initial stress state of the integration increment while using the adaptive sub-stepping to control the error. Both of the algorithms have been developed in classic elasto-plastic models but are still less reported for cyclic plasticity models. Manzari and Nour [7] first attempted to use an implicit algorithm in the bounding surface model for cyclic behaviours of soil. The results demonstrated the robustness of the implicit integration in 
the bounding surface model. However, one drawback of the model is the unrealistic description of cyclic loading because it is based on the fully isotropic hardening rule. Rouainia and Wood [8] presented an implicit return mapping integration in a modified bubble model based on a kinematic hardening rule, but it was only tested by a soil element. Borja et al. [9] used an implicit scheme to solve a two-surface model. However, the algorithm was run on the strain space in order to consider the nonlinear hyper-elasticity. Zhao et al. [15] argued that there were difficulties in the application of the implicit integration scheme to cyclic plasticity models and described the explicit integration of two complex constitutive models. However, they did not provide the performance of the algorithm in analysing the cyclic behaviour of the soil. Andrianopoulos et al. [16] proposed an explicit integration in the bounding surface model to analyse the earthquake liquefaction of noncohesive soils.

The accuracy, stability and efficiency of integration schemes are important issues in large-scale numerical simulation. However, comparative studies on the performance of the two integration algorithms in a complex cyclic plasticity model are rather limited. The conclusions from different researchers in solving boundary value problems are not uniform. Potts and Ganendra [17] compared the accuracy of return mapping implicit and sub-stepping explicit schemes in the Cam-clay model and stated that the sub-stepping algorithm was more accurate for a specific incremental size and for the analysis of a cavity expansion problem. Manzari and Prachathananukit [18] compared the closest point projection implicit integration with the sub-stepping explicit integration in a two-surface model and implemented them into finite element code. It was observed that for a relatively large strain increment, the implicit algorithm remained stable and accurate, while the explicit algorithm faced convergence difficulties. Sołowski et al. [19] ran both implicit and sub-stepping explicit integrations in the Barcelona basic model of unsaturated soil at a single stress point. However, it was concluded that for a larger strain increment, the implicit scheme offered faster convergence but might cause inaccurate computations. These findings highlight the importance of comparative studies on the accuracy, stability and efficiency of the two integration schemes.

The bounding surface plasticity model with a vanishing elastic region is more attractive for large-scale mathematical modelling related to cyclic loading because it is not necessary to address the evolvement of more than two yield surfaces (such as in the two-surface and multi-surface plasticity models [20-22]) and the smooth translation from nonlinear elastic to elasto-plastic behaviours. A recently developed anisotropic bounding surface model [23] has been shown to realistically present the stress-strain behaviours of the soils, including the cyclic shakedown and degradation. The present work is to implement the developed model with a vanishing elastic region [23] into a commercial finite element code with two integration schemes, i.e., the return mapping and sub-stepping integration schemes. However, there is no explicit current yield surface in the model to which the conventional implicit algorithm returns the stress state back or the sub-stepping integration corrects the drift of the stress state. Several necessary modifications should be made for both of the integration schemes. The performance, including the accuracy, robustness and efficiency of the two integration schemes, is investigated in detail both at the element level and in solving boundary value problems that involve monotonic and cyclic bearing behaviours of rigid footings on normally consolidated saturated clay.

\section{Outline of the anisotropic bounding surface model}

In this section, the anisotropic bounding surface plasticity model with a vanishing elastic region for saturated clay proposed by $\mathrm{Hu}$ et al. [23] is generalised to the multiaxial stress space. Within the framework of critical state soil mechanics, this model has been shown to accurately simulate important characteristics of saturated clay under cyclic loading such as initial anisotropy, reversal flow, cyclic shakedown and stiffness degradation by combining isotropic with kinematic hardening rules and adopting a rotational bounding surface. A brief description of the model is presented below.

In terms of notation, tensors are written in bold face characters to allow them to be easily distinguished from scalars. All of the presented stress quantities are effective. The symbol ':' denotes an inner product of two second-order tensors (e.g., $\mathbf{c}: \mathbf{d}=c_{i j} d_{i j}$ ) or a double contraction of the adjacent indices of tensors of rank two and higher (e.g., $\mathbf{C}: \boldsymbol{\varepsilon}^{e}=C_{i j k l} \varepsilon_{k l}^{e}$ ). The symbol ' $\otimes$ ' denotes the Kronecker product of two second-order tensors (e.g., $\mathbf{c} \otimes \mathbf{d}=c_{i j} d_{k l}$ ).

\subsection{Bounding surface formulation}

For the initial consolidation process, the form of the bounding surface in the model proposed by Hu et al. [23] is the same as the form adopted by Dafalias [24], which can be written in the conventional triaxial $p-q$ stress space as

$F=\bar{p}^{2}-\bar{p} p_{c}+\frac{(\bar{q}-\alpha \bar{p})^{2}}{M^{2}-\alpha^{2}}=0$

where $\bar{p}$ and $\bar{q}$ are mean effective and deviatoric stresses, respectively, and the superimposed bar indicates that the variables are related to the bounding surface; $M$ is the slope of the critical state line and equals $M_{e}$ for extension and $M_{c}$ for compression; $p_{c}$ and $\alpha$ define the size and inclination of the bounding surface, respectively, and their initial values are denoted by $p_{0}$ and $\alpha_{0}$. The concept of the model is shown graphically in Fig. 1 in the $p-q$ stress space.

The generalisation of Eq. (1) in the multiaxial stress space is obtained by standard methods $[25,26]$, as follows:

$F=\bar{p}^{2}-\bar{p} p_{c}+\frac{3}{2\left(M^{2}-\alpha^{2}\right)}[(\overline{\mathbf{s}}-\bar{p} \boldsymbol{\alpha}):(\overline{\mathbf{s}}-\bar{p} \boldsymbol{\alpha})]=0$

where $\overline{\mathbf{s}}$ and $\boldsymbol{\alpha}$ are deviatoric and anisotropic tensors, respectively, and $\alpha=\sqrt{\frac{3}{2} \alpha: \alpha}$ is a measure of the degree of soil anisotropy.

It can be seen from Eq. (2) that the bounding surface passes through the origin of the stress space. However, for the sequence shearing after the initial consolidation process, the model [23] has assumed that the bounding surface translates according to the kinematic hardening rule, which will be briefly explained in the following section (the details can be found in Ref. [23]). As a result, the endpoint of the bounding surface, which coincides with the origin of the stress space in the initial consolidation process, will translate to a new position in the stress space. We denote the endpoint as $\xi$ (Fig. 1). Hence, the translating bounding surface in the multiaxial stress space is expressed as

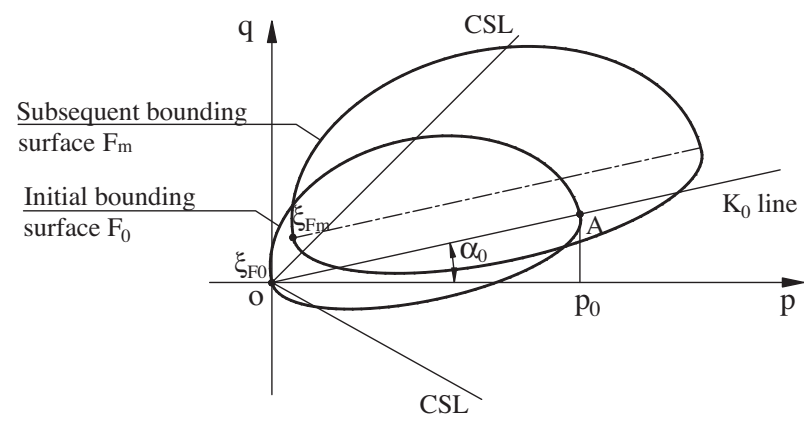

Fig. 1. Schematic of the rotational bounding surface in the $p-q$ space. 
$F_{m}=\hat{p}^{2}-\hat{p} p_{c}+\frac{3}{2\left(M^{2}-\alpha^{2}\right)} \hat{\mathbf{s}}: \hat{\mathbf{s}}=0$

with

$$
\left\{\begin{array}{l}
\hat{p}=\bar{p}-\xi_{p}^{(m)} \\
\hat{\mathbf{s}}=\overline{\mathbf{s}}-\xi_{s}^{(m)}-\left(\bar{p}-\xi_{p}^{(m)}\right) \boldsymbol{\alpha}
\end{array}\right.
$$

where $m$ is the ordinal number of specific loading events in which the stress path does not change direction. For example, $m=0$ means the initial consolidation process, and $m=1$ means the first loading or unloading event; stress tensors $\overline{\boldsymbol{\sigma}}$ and $\xi$ are expressed in terms of the volumetric and deviatoric components, which are defined as

$\bar{p}=\frac{1}{3} \operatorname{tr}(\overline{\boldsymbol{\sigma}}), \overline{\mathbf{s}}=\overline{\boldsymbol{\sigma}}-\bar{p} \mathbf{I}, \xi_{p}=\frac{1}{3} \operatorname{tr}(\boldsymbol{\xi}), \xi_{s}=\xi-\xi_{p} \mathbf{I}$

where $I$ is a second-rank identity tensor, and the subscripts $p$ and $s$ denote the volumetric and deviatoric components of a tensor, respectively.

\subsection{Hardening rules}

The characteristics of isotropic, kinematic hardening and even rotational hardening rules are included in the model proposed by Hu et al. [23]. In this section, the evolution for each of them is described briefly and generalised into multiaxial stress space.

\subsubsection{Isotropic hardening}

As in the original two-dimensional model [23], the scalar hardening variable $p_{c}$ controls isotropic hardening and determines the size of the bounding surface, which depends not only on the irreversible volumetric strain rate $\dot{\varepsilon}_{v}^{p}$ but also on the damage parameter $H$, which is related to the deviatoric plastic strain rate $\dot{\mathbf{e}}^{p}$ in the multiaxial stress space. The evolution equation for the size $p_{c}$ is given as

$$
\left\{\begin{array}{l}
p_{c}^{(n+1)}=p_{c}^{(n)} \exp \left(\chi_{0} \dot{\varepsilon}_{v}^{p}\right) H^{(n+1)} \\
H^{(n+1)}=H^{(n)} \exp \left(-\beta \dot{\varepsilon}_{A}\right)
\end{array}\right.
$$

with $\dot{\varepsilon}_{A}=\sqrt{\frac{2}{3} \dot{\mathbf{e}}^{p}: \dot{\mathbf{e}}^{p}}$ and $\chi_{0}=\frac{1+e_{0}}{\lambda-\kappa}$.

In Eq. (4), $p_{c}^{(n+1)}$ and $H^{(n+1)}$ denote the size and damage for the current load increment of the bounding surface, respectively; $p_{c}^{(n)}$ and $H^{(n)}$ denote the size and damage for the previous load increment of the bounding surface, respectively; $e_{0}$ denotes the void ratio after consolidation; and $\lambda$ and $\kappa$ are the slopes of the primary compression and swelling lines in the $e$ - $\ln p$ relations, respectively. It can be observed that $H$ decreases with the accumulated deviatoric plastic strain $\varepsilon_{A}$, which monotonically increases during the deformation process and is always positive. The decrease in $H$ induces a shrinkage of the bounding surface to reflect the degradation in stiffness and the reduction in strength. Further details of $H$ and $\varepsilon_{A}$ can be found in Ref. [23].

\subsubsection{Kinematic hardening}

The model assumes that the bounding surface isotropically hardens around the discrete homological centre (e.g., the stress reversal point). Once the stress reversal point occurs, the bounding surface should translate along the line that connects the stress reversal point and the image stress point. It can be seen that the kinematic hardening role arises from two parts. The first part is the movement of the bounding surface due to its isotropic hardening around the discrete homological centre. The second part is the translation of the bounding surface when the stress path changes direction. As a result, two cases should be noted in determining the location of the bounding surface.
Case 1: Upon the $(m+1)$ th loading event in which the stress path does not change direction, $F_{m+1}$ expands or contracts isotropically from the $n$th to the $(n+1)$ th loading substep. The endpoint $\xi$ of the current bounding surface $F_{m+1}$ is then expressed as follows:

$\left\{\begin{array}{c}\xi_{p(m+1)}^{(n+1)}=o_{p}^{(m+1)}+\left(\xi_{p(m+1)}^{(n)}-o_{p}^{(m+1)}\right) \frac{p_{c(m+1)}^{(n+1)}}{p_{c(m+1)}^{(n)}} \\ \xi_{s(m+1)}^{(n+1)}=\mathbf{o}_{s}^{(m+1)}+\left(\xi_{s(m+1)}^{(n)}-\mathbf{o}_{s}^{(m+1)}\right) \frac{p_{c(m+1)}^{(n+1)}}{p_{c(m+1)}^{(n)}}\end{array}\right.$

Case 2: When the stress path changes direction, $F_{m}$ translates along the line that connects the stress reversal point and the image point to form $F_{m+1}$. The endpoint $\xi$ of the current bounding surface $F_{m+1}$ is then expressed as

$$
\left\{\begin{array}{l}
\xi_{p}^{(m+1)}=\xi_{p}^{(m)}+(p-\bar{p}) \\
\xi_{s}^{(m+1)}=\xi_{s}^{(m)}+(\mathbf{s}-\mathbf{s})
\end{array}\right.
$$

where $(p, \mathbf{s})$ and $(\bar{p}, \overline{\mathbf{s}})$ are the newly formed stress reversal point and its image stress state, respectively; $\left(\xi_{p}^{(m+1)}, \xi_{s}^{(m+1)}\right),\left(\xi_{p(m+1)}^{(n)}, \xi_{s(m+1)}^{(n)}\right)$ and $\left(\xi_{p(m+1)}^{(n+1)}, \xi_{s(m+1)}^{(n+1)}\right)$ denote the endpoints of the bounding surfaces of the 0 th, $n$th and $(n+1)$ th loading substeps in the $(m+1)$ th loading event, respectively; $p_{c(m+1)}^{(n)}$ and $p_{c(m+1)}^{(n+1)}$ are the sizes of the bounding surfaces of the $n$th and $(n+1)$ th loading substeps in the $(m+1)$ th loading event, respectively; and $\left(o_{p}^{(m+1)}, \mathbf{o}_{s}^{(m+1)}\right)$ is the homological centre $\mathbf{o}$ of $F_{m+1}$, i.e., the coordinates of the last stress reversal point and the mapping centre. Details of the mapping centre and the image stress state are introduced in Section 2.3.

\subsubsection{Rotational hardening}

The model proposed by $\mathrm{Hu}$ et al. [23] has assumed that the initial anisotropy due to anisotropic consolidation is accounted for by adopting a rotational bounding surface at the start of shearing but without further rotation in the subsequent shearing, and the stress-induced anisotropy is considered by the above-mentioned kinematic hardening rule.

Similar to the definition adopted by Liang and Ma [26], Ling et al. [27] and Huang et al. [28], the initial anisotropic tensor $\alpha^{0}$ induced in the initial anisotropic consolidation is obtained through the deviatoric tensor $\mathbf{s}^{0}$ and the preconsolidated stress condition $p_{0}$ as

$\boldsymbol{\alpha}^{0}=\frac{\mathbf{s}^{0}}{p_{0}}$

For the $K_{0}$ consolidated samples, $K_{0}=\sigma_{3}^{0} / \sigma_{1}^{0}$, and the initial anisotropic tensors are given as follows:

$\alpha_{11}^{0}=\frac{2\left(1-K_{0}\right)}{1+2 K_{0}}, \quad \alpha_{22}^{0}=\alpha_{33}^{0}=\frac{K_{0}-1}{1+2 K_{0}}, \quad \alpha_{0}=3 \frac{1-K_{0}}{1+2 K_{0}}$

Hence, the inclination of the bounding surface $\alpha$, which is used to account for the initial anisotropy, can be obtained.

\subsection{Flow rule and mapping rule}

The plastic strain increments are governed by the associated flow rule

$\dot{\boldsymbol{\varepsilon}}^{p}=\langle\Lambda\rangle \overline{\mathbf{n}}, \Lambda=\frac{\overline{\mathbf{n}}: \dot{\boldsymbol{\sigma}}}{K_{p}}=\frac{\overline{\mathbf{n}}: \overline{\boldsymbol{\sigma}}}{\overline{K_{p}}}$

where $\Lambda$ is the loading index; \langle\rangle is the symbol of Macauly brackets; $K_{p}$ and $\overline{K_{p}}$ are the plastic moduli at the current and image stress points, respectively; and $\overline{\mathbf{n}}=\{\partial F / \partial \bar{p} \mathbf{I}, \partial F / \partial \overline{\mathbf{s}}\}=\left\{\bar{n}_{p} \mathbf{I}, \overline{\mathbf{n}}_{s}\right\}$ denotes the tensor of the stress gradient on the bounding surface at the current stress state.

To define the image point at the bounding surface in a simple way, the radial mapping rule proposed by Dafalias [24] is adopted. 
The image stress point $\overline{\boldsymbol{\sigma}}$ is defined by projecting the radial line that connects the mapping centre $\mathbf{o}$ and the current stress state $\boldsymbol{\sigma}$ onto the bounding surface. The radial mapping rule is expressed by

$\overline{\boldsymbol{\sigma}}=\mathbf{o}+b(\boldsymbol{\sigma}-\mathbf{o})$

where $b$ is the ratio of the distance between the mapping origin and the image stress point to the distance between the mapping origin and the current stress state, which can be determined by substituting Eq. (8) into the analytical expression of $F=0$ (see the expression in Section 3.2). The mapping centre $\mathbf{o}$ is translated to capture the plastic flow in the reverse loading [23] and can be determined by the following equation:

$\left(o_{p, n+1}, \mathbf{o}_{s, n+1}\right)=\left\{\begin{array}{l}\left(o_{p, n}, \mathbf{o}_{s, n}\right) \text { if } \overline{\mathbf{n}}_{n}: \boldsymbol{\sigma}_{n+1} \geqslant 0 \\ \left(p_{n}, \mathbf{s}_{n}\right) \text { if } \overline{\mathbf{n}}_{n}: \boldsymbol{\sigma}_{n+1}<0\end{array}\right.$

in which the subscripts $n$ and $n+1$ refer to the previous and current load steps, respectively.

\subsection{Elastic and plastic moduli}

The elastic components of deviatoric and volumetric strain rates are obtained following the standard relationships

$\dot{\mathbf{e}}^{e}=\frac{\dot{\mathbf{s}}}{2 G}, \quad \dot{\varepsilon}_{v}^{e}=\frac{\dot{p}}{K}$

Similar to the critical state models $[12,15,18]$, the tangential bulk and shear moduli in the present model are assumed to depend linearly on the mean effective stress and to satisfy the following equation

$G=\frac{3(1-2 v)}{2(1+v)} K$

where $v$ is the constant Poisson's ratio.

The plastic component of the strain rate is based on the form given to the plastic modulus (see Eq. (7)). The plastic modulus can be given by the consistency condition on the bounding surface as

$\overline{K_{p}}=\hat{p} p_{c}\left(\bar{n}_{p} \chi_{0}-\frac{2 \beta}{M^{2}-\alpha^{2}} \sqrt{\frac{3}{2} \hat{\mathbf{s}}: \hat{\mathbf{s}}}\right)$

The plastic modulus of the current stress state can be obtained by employing the interpolation rule as [23]

$K_{p}=\overline{K_{p}}+\rho\left(\bar{p}, \overline{\mathbf{s}}, \varepsilon_{v}^{p}, \mathbf{e}^{p}\right)(b-1)^{\gamma}$

with $\rho\left(\bar{p}, \overline{\mathbf{s}}, \varepsilon_{v}^{p}, \mathbf{e}^{p}\right)= \begin{cases}\left|K_{m}-\overline{K_{p}}\right| & \text { for first loading } \\ \left|\varsigma_{u} K_{m}-\overline{K_{p}}\right| & \text { for unloading } \\ \left|\varsigma_{r} K_{m}-\overline{K_{p}}\right| & \text { for reloading }\end{cases}$

where $K_{m}$ is the bounding plastic modulus on the last stress reversal, and

$\frac{\zeta_{u}}{\zeta_{r}}=\left(\frac{M_{c}}{M_{e}}\right)^{2}\left(1+\bar{n}_{p} / \eta\right)$

in which $\gamma, \zeta_{r}$ and $\eta$ are positive model parameters, whose physical meaning and calibration are given in the literature [23].

\section{A return mapping integration for the anisotropic bounding surface model}

In this section, a conventional implicit integration scheme based on the return mapping algorithm [6] is modified and developed into the anisotropic bounding surface model described above. Then, the model is implemented into a commercially available finite element code.
It is known that in the conventional elasto-plastic model, the return mapping algorithm consists of two basic sequences, namely the elastic trial and the plastic corrector depending on whether the elastic trial stress falls inside or outside the yield surface. However, as mentioned earlier, the elastic region is reduced to a point, and there is no explicit current yield surface in the present bounding surface model. Hence, several modifications of the return mapping algorithm are necessary to make it useful to this type of single bounding surface model.

\subsection{Elastic trial}

In the process of an elastic trial, the plastic response remains constant and equals the respective value at the previous increment (say, $n$ ). For the initial iteration number $k=0$,

$$
\left\{\begin{array}{l}
\Delta \varepsilon_{v, n+1}^{p(0)}=\Delta \mathbf{e}_{n+1}^{p(0)}=\Delta H_{n+1}^{(0)}=0 \\
p_{c, n+1}^{(0)}=p_{c, n}, \xi_{n+1}^{(0)}=\xi_{n}, \mathbf{o}_{n+1}^{(0)}=\mathbf{o}_{n}
\end{array}\right.
$$

where the subscript $n+1$ indicates the variables that are related to the current increment. The trial stress is obtained from the following equations:

$p_{n+1}^{(0)}=p_{n} \exp \left(\frac{1+e_{0}}{\boldsymbol{\kappa}} \Delta \varepsilon_{v, n+1}\right), \mathbf{s}_{n+1}^{(0)}=\mathbf{s}_{n}+2 G_{n+1}^{(0)} \Delta \mathbf{e}_{n+1}$

Integrating Eq. (10a) with $p$ and $\Delta \varepsilon_{v}^{e}$, the secant bulk modulus can be derived as

$K_{n+1}^{(0)}=\frac{p_{n} \exp \left[\frac{1+e_{0}}{\kappa} \Delta \varepsilon_{v, n+1}\right]-p_{n}}{\Delta \varepsilon_{v, n+1}}$

and the secant shear modulus can be expressed as

$G_{n+1}^{(0)}=\frac{3(1-2 v)}{2(1+v)} K_{n+1}^{(0)}$

It should be noted that, in the case $\Delta \varepsilon_{v, n+1}=0$, we have

$p_{n+1}^{(0)}=p_{n}, \mathbf{s}_{n+1}^{(0)}=\mathbf{s}_{n}+2 \frac{3(1-2 v)}{2(1+v)} p_{n} \frac{1+e_{0}}{\kappa} \Delta \mathbf{e}_{n+1}$

\subsection{Plastic correction}

In the process of the plastic correction, two characteristics of the bounding surface model should be noted. First, due to the elastic region vanishing in the bounding surface model, the plastic flow occurs immediately for any stress increment within the bounding surface. Second, in contrast to the conventional plastic models that consider the unloading elastic, the present model can capture the reverse plastic flow by adopting the discrete stress reversal point as the mapping origin in the radial mapping rule. Hence, two modifications of the conventional return mapping algorithm are correspondingly made. For the first characteristic, the trial image stress state is mapped onto the bounding surface instead of mapping back or correcting the stress state to the yield surface. In fact, similar to the work by Borja et al. [9], the condition of consistency on the bounding surface implies the condition of consistency on the yield surface (referring to the current stress point). The details can be found in Appendix A. For the second characteristic, the loading-unloading criterion is checked to distinguish the mapping origin and the homological centre and then to determine the location of the bounding surface to obtain the trial image stress point, rather than judging whether the stress point is inside the yield surface.

To map the image stress state back onto the bounding surface, it is necessary to meet all of the incremental constitutive relations, as follows: 


$$
\begin{aligned}
& p_{n+1}=p_{n} \exp \left[\frac{1+e_{0}}{\kappa}\left(\Delta \varepsilon_{v, n+1}-\Delta \varepsilon_{v, n+1}^{p}\right)\right] \\
& \Delta \varepsilon_{v, n+1}^{p}=\Lambda_{n+1} \bar{n}_{p, n+1} \\
& \mathbf{s}_{n+1}=\mathbf{s}_{n}+2 G_{n+1}\left(\Delta \mathbf{e}_{n+1}-\Delta \mathbf{e}_{n+1}^{p}\right) \\
& \Delta \mathbf{e}_{n+1}^{p}=\Lambda_{n+1} \overline{\mathbf{n}}_{s, n+1} \\
& p_{c, n+1}=p_{c, n} \exp \left(\chi_{0} \Delta \varepsilon_{v, n+1}^{p}\right) H_{n+1} \\
& H_{n+1}=H_{n} \exp \left(-\beta \sqrt{\frac{2}{3} \Delta \mathbf{e}_{n+1}^{p}: \Delta \mathbf{e}_{n+1}^{p}}\right) \\
& \left(\hat{p}_{n+1}\right)^{2}-\hat{p}_{n+1} p_{c, n+1}+\frac{3}{2\left(M^{2}-\alpha^{2}\right)} \hat{\mathbf{s}}_{n+1}: \hat{\mathbf{s}}_{n+1}=0 \\
& b_{n+1}=b_{n}+\Lambda_{n+1} \frac{\bar{K}_{p, n+1}-b_{n+1} K_{p, n+1}}{A_{n+1}} \\
& \bar{K}_{p, n+1}=\hat{p}_{n+1} p_{c, n+1}\left(\chi_{0} \bar{n}_{p, n+1}-\frac{2 \beta}{M^{2}-\alpha^{2}} \sqrt{\frac{3}{2} \hat{\mathbf{s}}_{n+1}: \hat{\mathbf{s}}_{n+1}}\right)
\end{aligned}
$$

In Eq. (14h),

$$
\begin{aligned}
A= & p_{c}\left(p-o_{p}\right)+2\left(p-o_{p}\right)\left(\xi_{p}-o_{p}\right)+\frac{3}{\left(M^{2}-\alpha^{2}\right)}\left[\left(\mathbf{s}-\mathbf{o}_{s}\right)\right. \\
& \left.-\left(p-o_{p}\right) \boldsymbol{\alpha}\right]:\left[\left(\xi_{s}-\mathbf{o}_{s}\right)-\left(\xi_{p}-o_{p}\right) \boldsymbol{\alpha}\right]
\end{aligned}
$$

Eqs. (14a) and (14c) represent the elastic strains and their relations to the stress states; Eqs. (14b) and (14d) present the flow rule; Eqs. (14e) and (14f) represent the isotropic hardening laws; and Eq. (14g) represents the condition of consistency on the bounding surface. It is observed that the condition of consistency needs the updated image stress point. As a result, the above equations include not only the updated stress, the flow rule, the hardening laws and the condition of consistency such as in the conventional methods but also the mapping rule (e.g., Eq. (14h)), to obtain the image point and Eq. (14i) to furnish the additional equation that is necessary for solving the set of equations. Thus, Eq. (14) constitutes a system of nonlinear implicit equations that must be solved simultaneously and iteratively using the Newton-Raphson procedure. Note that Eqs. (5) and (9) must be used to obtain the locations of the bounding surface and the mapping origin (e.g., $\xi$ and o). However, it can be seen from Eqs. (5) and (9) that $\xi$ is not involved in the iterative procedure because the kinematic hardening rule is discrete and related only to the previous location, the newest stress reversal and the current size of the bounding surface.

\subsection{Stress updating procedure}

A full stress updating procedure is given in Table 1, where the superscript $k$ refers to the local iteration number, and the subscripts $n$ and $n+1$ denote the previous and current load steps, respectively. It should be noted that, in order to compare the performance of the two integrations at the same level of error, the residual tolerance in the implicit scheme and the specified tolerance for the explicit sub-stepping integration (i.e., STOL in Table 2) are all set to $10^{-5}$, which lies in the typical range of values suggested by Sloan et al. [12] and Zhao et al. [15].

\subsection{Consistent tangent operator}

To maintain the main advantage of the implicit integration scheme, i.e., the quadratic convergence of the Newton iteration, it is necessary to use the consistent tangent operator in the solution of the global finite element equation. Here, consistency means that the stress increment calculated by the tangent modulus operating on the strain increment matches the stress increment calculated by the integration procedure to first-order [29].
The general form of the consistent tangent operator $\mathbf{C}_{n+1}^{k}$ is derived as

$\mathbf{C}_{n+1}^{k}=\frac{\partial \Delta p_{n+1}^{k}}{\partial \Delta \boldsymbol{\varepsilon}_{n+1}^{k}} \otimes \mathbf{I}+\frac{\partial \Delta \mathbf{s}_{n+1}^{k}}{\partial \Delta \boldsymbol{\varepsilon}_{n+1}^{k}}$

To evaluate the consistent tangent operator, Eqs. 14a, 14c, and 14e, which directly relate to $p, \mathbf{s}, \varepsilon_{\nu}$ and $\mathbf{e}$, are written in the differential form, as follows:

$\left\{\begin{array}{l}\Delta p=K\left(\Delta \varepsilon_{v}-\bar{n}_{p} \Delta \Lambda-\Delta \bar{n}_{p} \Lambda\right) \\ \Delta \mathbf{s}=2 G\left(\Delta \mathbf{e}-\overline{\mathbf{n}}_{s} \Delta \Lambda-\Delta \overline{\mathbf{n}}_{s} \Lambda\right) \\ \Delta p_{c}=-\frac{p_{c} \chi_{0}}{K} \Delta p+\frac{\beta p_{c}}{3 G} \frac{\Delta \mathbf{e}^{p}}{\Delta \varepsilon_{A}}: \Delta \mathbf{s}+p_{c} \chi_{0} \Delta \varepsilon_{v}-\frac{2 \beta p_{c}}{3 \Delta \varepsilon_{A}} \Delta \mathbf{e}^{p}: \Delta \mathbf{e}\end{array}\right.$

in which $\left\{\begin{array}{l}\bar{n}_{p}=2 \hat{p}-p_{c}-\frac{3 \alpha \cdot \hat{\mathbf{s}}}{M^{2}-\alpha^{2}} \\ \overline{\mathbf{n}}_{s}=\frac{3 \hat{\mathbf{s}}}{\boldsymbol{M}^{2}-\alpha^{2}}\end{array}\right.$

It can be seen that the unknowns $\left(\Delta \bar{n}_{p}, \Delta \overline{\mathbf{n}}_{s}\right)$ and $\Delta \Lambda$ are related to Eqs. (14g) and (14h), which reflect the mapping rule and the consistency condition, respectively. As a result, Eqs. (14g) and (14h) should be differentiated with respect to $\boldsymbol{\varepsilon}$. The additional derivative of the plastic modulus, Eq. (14i), furnishes the system of linear equations. Thus, there are a total of six independent unknowns $\left(\Delta p, \Delta \mathbf{s}, \Delta p_{c}, \Delta b, \Delta \Lambda, \Delta \bar{K}_{p}\right)$ and six linear equations. The consistent tangent operator can then be determined, and the details are presented in Appendix B.

\section{A sub-stepping integration for the anisotropic bounding surface model}

In addition to the return mapping integration approach, the explicit integration scheme based on the forward modified Euler method with automatic sub-stepping and error control [12] is also adopted to integrate the rate form of stress-strain relations in the present model.

In general, the explicit algorithm involves three parts [12]: (1) locating the yield surface intersection with the elastic trial stress path to compute the portion of the given strain increment that corresponds to the plastic deformation; (2) integrating the rate equations of the stress and internal variables via a second-order forward modified Euler scheme with sub-stepping and error control; and (3) correcting the drift of the yield surface at the end of the successful sub-increment. Similar to the situation in the implicit integration, modifications are still required when applying the scheme to the bounding surface model without a yield surface. First, it is not necessary to perform the first part to define the portion of the plastic strain because the elastic region is reduced to a point, which means that the total given strain increment causes the plastic flow. Second, in the third part, instead of correcting the stress state to the yield surface, the image stress point is enforced to lie on the bounding surface at the end of the successful sub-increment. The details are described in Section 4.2 .

\subsection{General formulations}

For a given strain increment, we have

$\dot{\boldsymbol{\sigma}}=\mathbf{D}_{\mathbf{e}}: \dot{\boldsymbol{\varepsilon}}^{e}=\mathbf{D}_{\mathbf{e}}:(\dot{\boldsymbol{\varepsilon}}-\Lambda \overline{\mathbf{n}})$

where $\mathbf{D}_{\mathbf{e}}$ is the elastic stiffness matrix. By decomposing the right terms in Eq. (17a) into volumetric and deviatoric components, Eq. (17a) can be written as

$\dot{\boldsymbol{\sigma}}=2 G \dot{\mathbf{e}}+K \dot{\boldsymbol{\varepsilon}}_{v} \mathbf{I}-\langle\Lambda\rangle\left(2 G \overline{\mathbf{n}}_{s}+K \bar{n}_{p} \mathbf{I}\right)$

Substituting Eq. (17b) into Eq. (7), the loading index is expressed as 
Table 1

Stress updating procedure of the return mapping scheme.

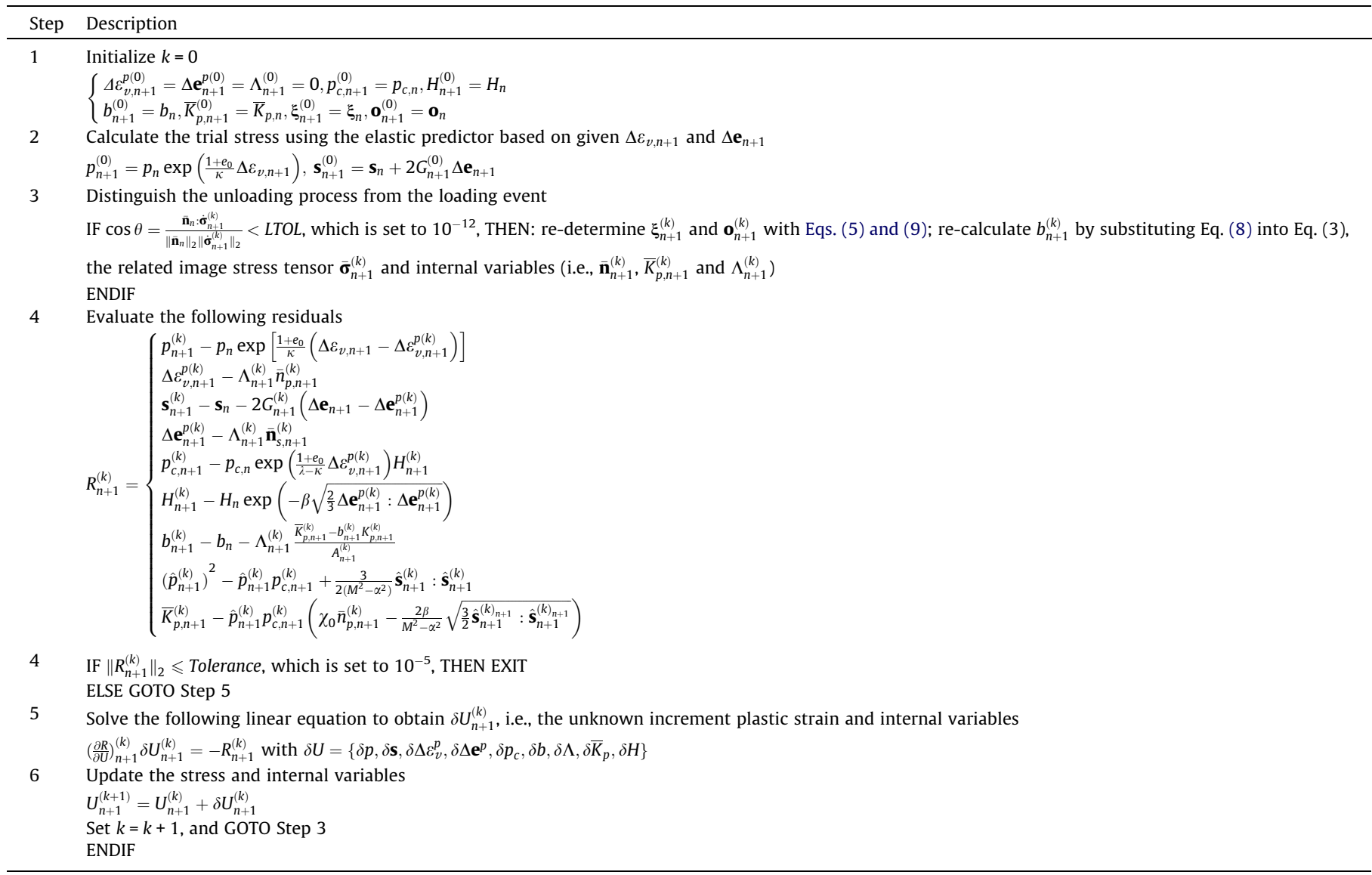

$\Lambda=\frac{\overline{\mathbf{n}}: \dot{\boldsymbol{\sigma}}}{K_{p}}=\frac{2 G \overline{\mathbf{n}}_{s}: \dot{\mathbf{e}}+K \bar{n}_{p} \dot{\varepsilon}_{v}}{K_{p}+2 G \overline{\mathbf{n}}_{s}: \overline{\mathbf{n}}_{s}+K\left(\bar{n}_{p}\right)^{2}}$

\subsection{Stress integration procedure with the sub-stepping algorithm}

The second part of the explicit algorithm, i.e., the integration of the rate equations, works in the following way: once a strain increment is given, the set of the stress increment and the increments of the internal variables can be calculated based on the current stress state. Then, update the stress and internal variables and use them to obtain another set of increments of the stress and internal variables. If the difference between two sets of solutions cannot satisfy the prescribed tolerance, the strain increment is subdivided automatically into a smaller sub-increment. For a given strain increment, the integration is accomplished in one or more sub-increments. The procedure of the sub-stepping integration for the anisotropic bounding surface model is listed in Table 2 . We define the pseudo time $T$ $(0 \leqslant T \leqslant 1)$ for each strain increment $\dot{\boldsymbol{\varepsilon}}$ over a time step $\left[t_{n}, t_{n+1}\right]$. At the same time, the sub-increment is denoted by $\dot{\boldsymbol{\varepsilon}}^{s}$ with a pseudo time $\Delta T_{m}\left(0 \leqslant \Delta T_{m} \leqslant 1\right)$, in which the subscript $n$ and the superscript $m$ denote the numbers of increments and sub-increments, respectively, and the superscript $s$ refers to the sub-increment.

In Step 2 of Table 2, the total given strain increment is used to determine the strain sub-increment. In Step 3, the unloading-loading criterion is checked to determine the locations of the bounding surface and the mapping rule because they are different for the unloading and loading events. In Step 8, similar to correcting the drift of the stress to the yield surface in the conventional sub-stepping algorithm, the image stress point is enforced to be on the bounding surface. To keep the total strain increment unchanged, a consistency correction scheme similar to the scheme proposed by Sloan et al. [12] is adopted. This choice was made because the consistency of the bounding surface ensures the condition of consistency on the yield surface (details can be seen in Appendix A). In Step 10, the coefficients 0.9 and 1.1 act merely as safety factors, which are adjustable to suit the specific models [12]. According to the suggestion by Sloan et al. [12] and Zhao et al. [15], the bounding surface tolerance (FTOL) is set to $10^{-9}$.

\subsection{The continuum tangent operator}

The Jacobian stiffness matrix must be given and updated after the successful stress integration, and then, must be passed to the global finite element routine. Here, the Jacobian stiffness matrix is the continuum tangent operator and is derived following the same procedure in solving the classical elasto-plastic modulus, i.e., substituting the consistency, flow rule and hardening laws into the incremental relations between the stress and strain. The continuum Jacobian stiffness matrix is represented as

$\mathbf{D}_{\mathbf{e p}}=\mathbf{D}_{\mathbf{e}}-\frac{\left(\mathbf{D}_{\mathbf{e}}: \overline{\mathbf{n}}\right) \otimes\left(\overline{\mathbf{n}}: \mathbf{D}_{\mathbf{e}}\right)}{K_{p}+\overline{\mathbf{n}}: \mathbf{D}_{\mathbf{e}}: \overline{\mathbf{n}}}$

\section{Performance of the integration schemes}

In this section, the generalised three-dimensional bounding surface plasticity model with a vanishing elastic region is implemented into the commercial software ABAQUS. Then, the performance of the implicit and explicit integration schemes described above, i.e., the accuracy, stability and efficiency, is assessed 
Table 2

Procedure of the sub-stepping integration scheme in the model.

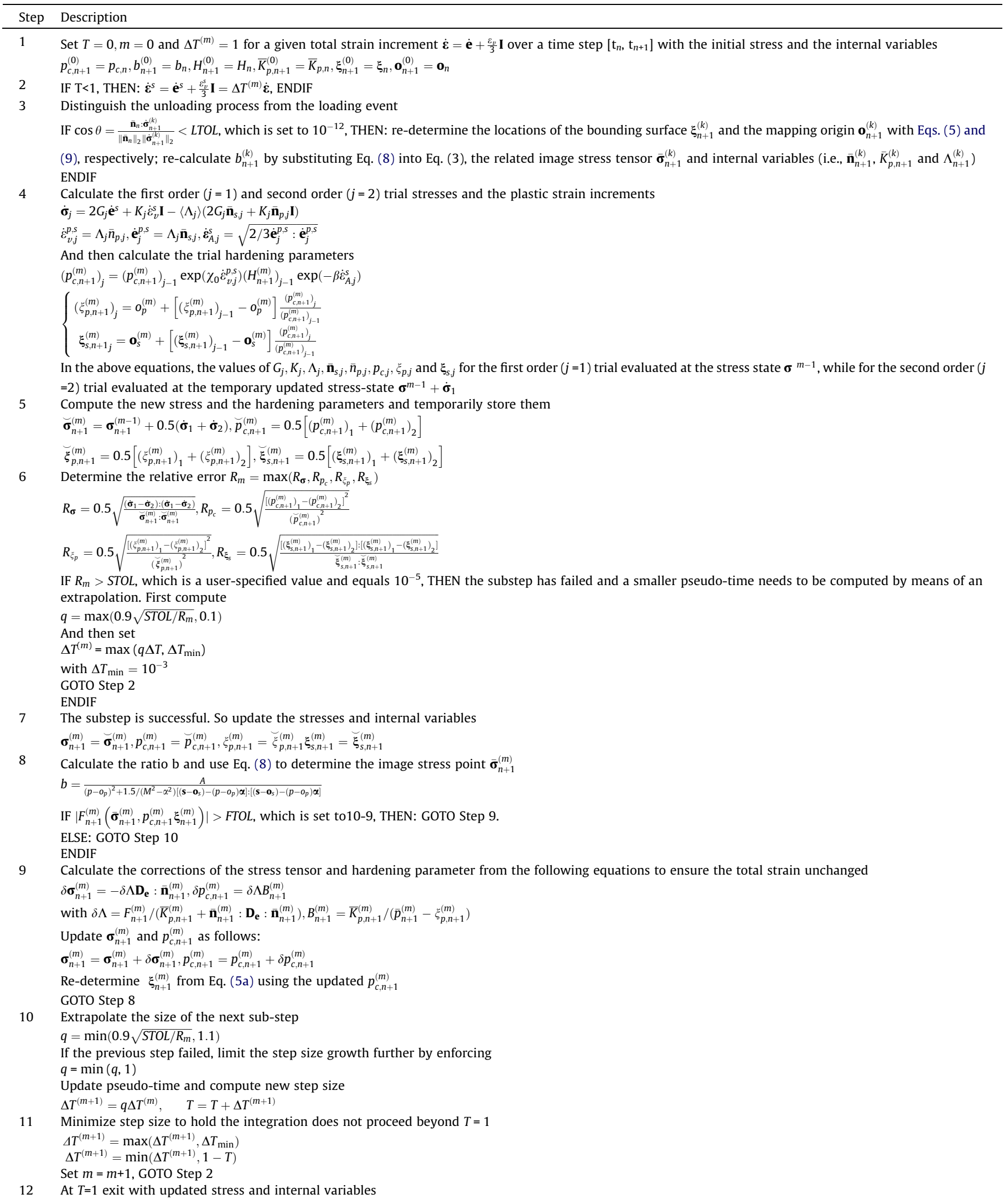

through simulating the conventional laboratory tests, including the triaxial shear tests, the stress-controlled cyclic triaxial tests and the strain-controlled cyclic triaxial tests, and performing coupled analyses of rigid footings on saturated clay under both monotonic and cyclic loading. The element types used in Sections 5.1-5.5 include the 8-node trilinear displacement element and the 
pore-pressure element C3D8P, and the element types used in Section 5.6 include the 8-node biquadratic displacement element and the pore-pressure element CPE8P [30].

\subsection{Conventional triaxial shear test for $K_{0}$-consolidated clay}

The first problem of interest involves an undrained conventional triaxial test on a normally consolidated clay. The model parameters reported by Stipho [31] for Kaolin clay are used and listed in Table 3, which were also used for calibrating the constitutive models by Liang and Ma [26] and Ling et al. [27]. Triaxial shear tests, including compression and extension tests on both isotropically and anisotropically consolidated specimens, are simulated using a cubical element with the size $102 \mathrm{~mm} \times 102 \mathrm{~mm} \times 102 \mathrm{~mm}$. The initial conditions for the two cases $\left(K_{0}=1\right.$ and $\left.K_{0}=0.67\right)$ are the initial void ratio $e_{0}=1.6$ and the mean effective stress $p_{0}=210 \mathrm{kPa}$ (e.g., $\sigma_{1}=$ $\sigma_{2}=\sigma_{3}=210 \mathrm{kPa}$ ) or $p_{0}=163 \mathrm{kPa}$ (e.g., $\sigma_{1}=210 \mathrm{kPa}, \sigma_{1}=210$, $\sigma_{2}=\sigma_{3}=140 \mathrm{kPa}$ ). The element is fixed at the bottom with constant lateral pressure, and then, it is subjected to the axial strain at the top with a magnitude of $10 \%$ and $-10 \%$ for compression and extension, respectively.

The tests are performed with different strain increments to a maximum axial strain of $10 \%$. Fig. 2 shows the stress-strain response, the effective stress path and the pore pressure-strain curve of both the compression and extension tests when using the return mapping algorithm for $K_{0}=1$. As seen in Fig. 2, all of the simulations are close to each other, even at a relatively larger strain increment of $2 \%$. This finding demonstrates the stability and accuracy of the implicit integration. The behaviour of the combination of the consistent tangent operator and the Newton-Raphson procedure for local iteration at a moderate strain increment of $0.2 \%$ is demonstrated in Table 4, in which the residual norms are shown for typical load increments. This result clearly indicates that the quadratic rate of asymptotic convergence is achieved.

Fig. 3 shows the corresponding simulation of the triaxial extension test for $K_{0}=0.67$ using the sub-stepping algorithm. Similar to the return mapping rule, the sub-stepping integration provides a solution with reasonable accuracy. Here, the convergence problem occurs when imposing the axial strain of $10 \%$ in 50 increments. Fig. 4 presents the comparison between the triaxial compression data and the simulation results for $K_{0}=0.67$ by using the implicit and explicit integrations with the same increment size. It is observed that all of the simulations that use the implicit and explicit algorithms generally match with the experimental data. The difference induced by the two approaches is not significant. However, the sub-stepping algorithm predicts a higher shear strength at the same increment size. In fact, Sołowski et al. [19] have reported a similar phenomenon and noted that the difference between the implicit and explicit schemes reaches 30\% for a Gaussian integration point in a triaxial stress state.

Table 3

Values of the model parameters.

\begin{tabular}{lllll}
\hline Parameters & $\begin{array}{l}\text { Kaolin } \\
\text { clay }\end{array}$ & $\begin{array}{l}\text { Newfield } \\
\text { clay }\end{array}$ & $\begin{array}{l}\text { Marine plastic } \\
\text { clay }\end{array}$ & $\begin{array}{l}\text { Saturated } \\
\text { clay }\end{array}$ \\
\hline Traditional & & & & \\
$e_{0}$ & 1.1 & 0.62 & 2.422 & 0.929 \\
$v$ & 0.20 & 0.15 & 0.30 & 0.30 \\
$M_{e}$ & 0.846 & 0.83 & 1.456 & 1.46 \\
$M_{c}$ & 1.178 & 1.14 & 1.560 & 1.56 \\
$\kappa$ & 0.05 & 0.0108 & 0.057 & 0.17 \\
$\lambda$ & 0.14 & 0.0508 & 0.349 & 0.05 \\
Hardening & & & & \\
$\gamma$ & 2 & 1.5 & 2 & 2 \\
$\zeta_{r}$ & - & 3.4 & 2 & 5 \\
$\eta$ & & 120 & 100 & 100 \\
$\beta$ & & - & 0.005 & - \\
\hline
\end{tabular}

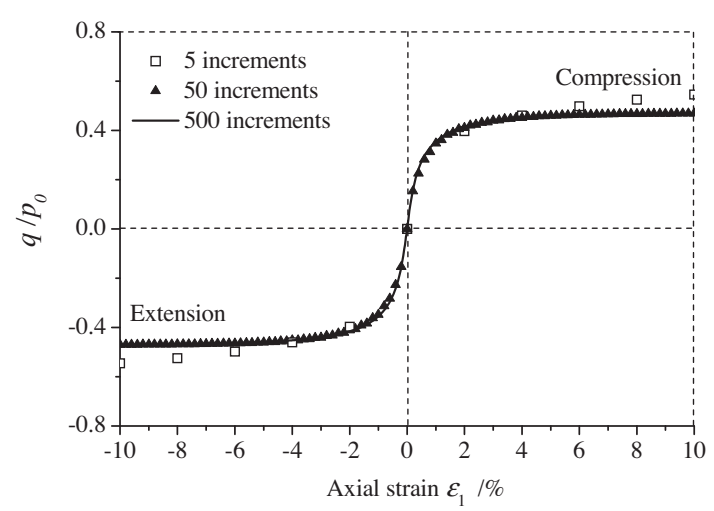

(a) Stress-strain relations

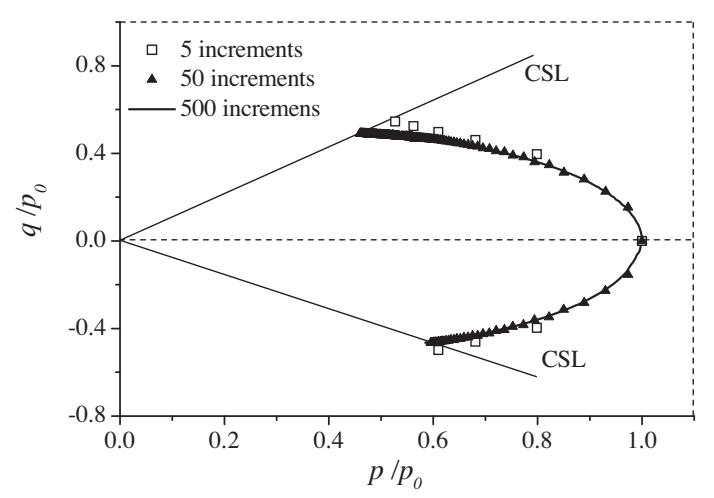

(b) Effective stress path

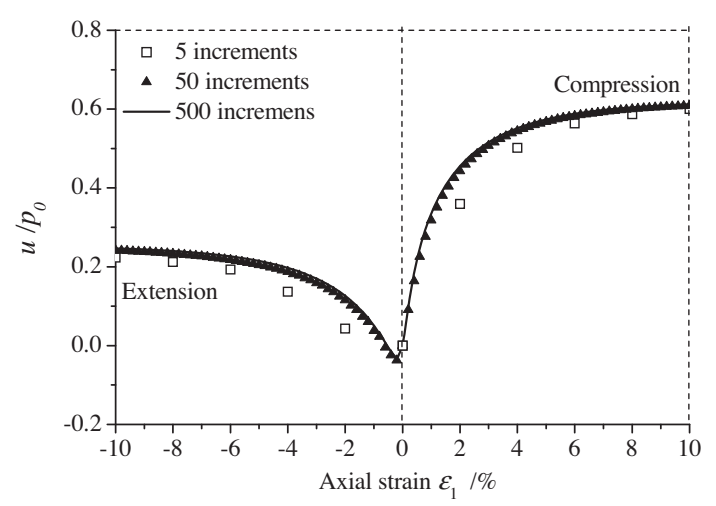

(c) Pore pressure-strain curve

Fig. 2. Model simulation by the return mapping in undrained triaxial tests with variable magnitude of strain increment.

\subsection{Iso-error maps}

To further assess the accuracy of the two integrations in the present model, a more systematic error analysis is performed in the manner described by Simo and Hughes [6]. The relative error $\delta$ defined by Borja et al. [9] is expressed as

$\delta=\frac{\sqrt{\left(\boldsymbol{\sigma}-\boldsymbol{\sigma}^{*}\right):\left(\boldsymbol{\sigma}-\boldsymbol{\sigma}^{*}\right)}}{\sqrt{\boldsymbol{\sigma}^{*}: \boldsymbol{\sigma}^{*}}} \times 100 \%$

where $\boldsymbol{\sigma}$ is the result obtained by applying the algorithms; and $\boldsymbol{\sigma}^{*}$ is the exact solution that corresponds to the specified strain increment, which decreases as it produces no significant change in the results.

Here, iso-error maps are established by first constructing a stress state after isotropical consolidation for Kaolin clay at an 
Table 4

Iteration process of the return mapping integration (Kaolin clay, $K_{0}=1,50$ increments): residual norms for typical load increments at integration point 3.

\begin{tabular}{lllll}
\hline Iteration & Increment 1 & Increment 20 & Increment 40 & Increment 60 \\
\hline 1 & $1.2951 \mathrm{E}+3$ & $4.9837 \mathrm{E}+3$ & $4.9072 \mathrm{E}+3$ & $4.9054 \mathrm{E}+3$ \\
2 & $3.113 \mathrm{E}+1$ & $2.9041 \mathrm{E}+2$ & $2.8171 \mathrm{E}+2$ & $2.8151 \mathrm{E}+2$ \\
3 & $3.629 \mathrm{E}-1$ & $3.7213 \mathrm{E}+0$ & $3.6824 \mathrm{E}+0$ & $3.6815 \mathrm{E}+0$ \\
4 & $2.684 \mathrm{E}-3$ & $7.1423 \mathrm{E}-2$ & $7.0854 \mathrm{E}-2$ & $7.0840 \mathrm{E}-2$ \\
5 & $4.137 \mathrm{E}-6$ & $1.3622 \mathrm{E}-4$ & $1.3574 \mathrm{E}-4$ & $1.3572 \mathrm{E}-4$ \\
6 & - & $4.9551 \mathrm{E}-7$ & $4.9825 \mathrm{E}-7$ & $4.9829 \mathrm{E}-7$ \\
\hline
\end{tabular}

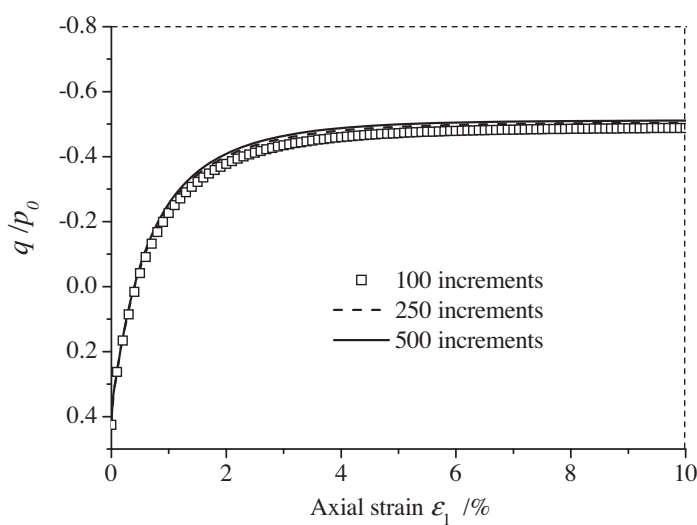

(a) Stress-strain relations

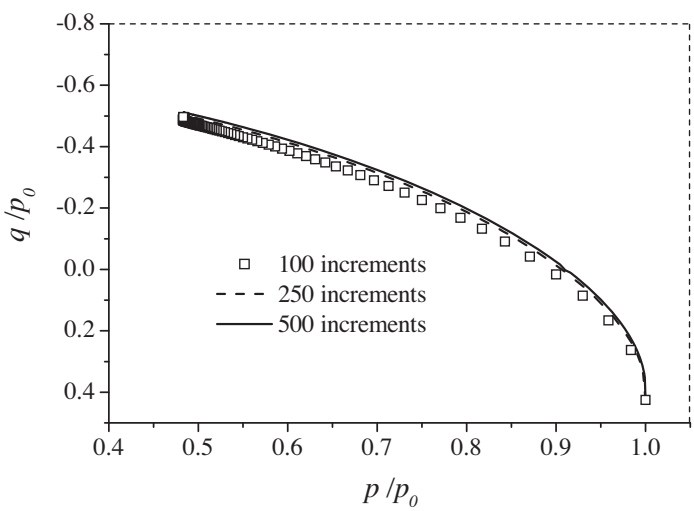

(b) Effective stress path

Fig. 3. Model simulation by the sub-stepping in undrained triaxial tests with variable magnitude of strain increment.

element level, which is described in Section 5.1, then applying the axial strain at a magnitude of $10^{-4}$ in the direction of the $\sigma_{1}$ axis (i.e., the $z$-axis) to bring the soil element into the triaxial compression state and then to impose a sequence of specified strain increments by simultaneously applying displacements in the directions of $\sigma_{1}$ and $\sigma_{2}$ (i.e., the $x$-axis). For each strain probe, the exact solution is obtained by dividing the desired displacement increment into 1000 sub-increments. The model constants are listed in Table 3. In the sub-stepping integration, the local stress tolerance (STOL) and the bounding surface tolerance (FTOL) are set to $10^{-5}$ and $10^{-9}$, respectively.

Figs. 5 and 6 present the iso-error maps for the loading from the initial isotropic stress state using the return mapping and substepping algorithms, respectively. It is observed that, in the strain increment range of $0-1 \%$, the relative error becomes larger with an increasing strain increment both for the return mapping and the sub-stepping algorithms. This result does not coincide with

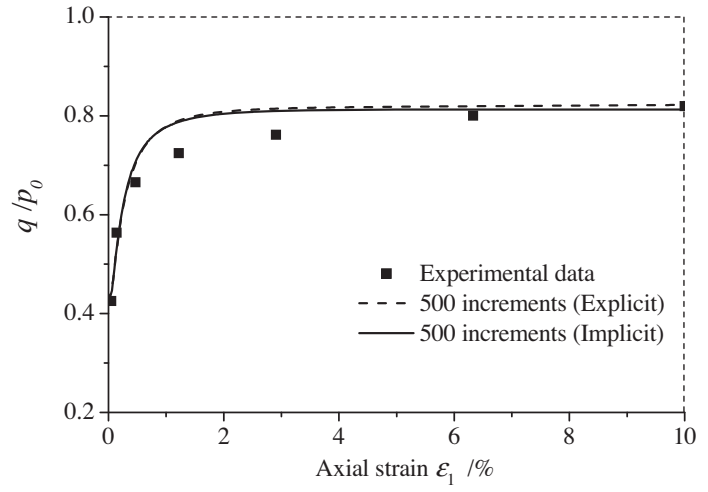

(a) Stress-strain relations

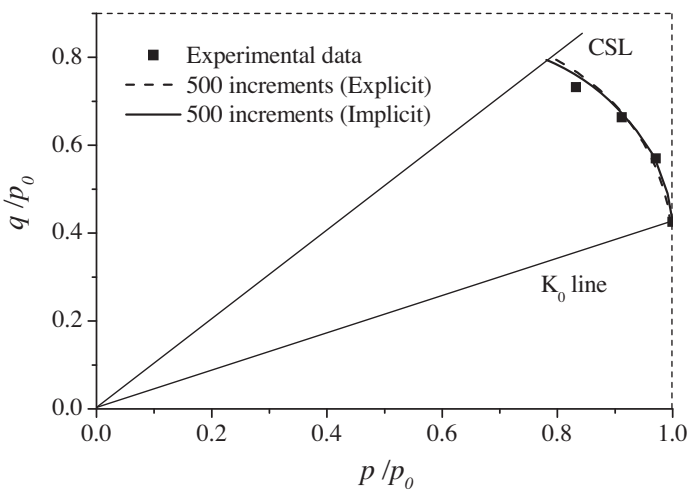

(b) Effective stress path

Fig. 4. Comparison between the predicted results and experimental data [31].

the result from Potts and Ganendra [17], in which the errors from both stress point algorithms increase first and then decrease with the strain increment size. The possible reason is that a relatively large-scale strain increment was adopted by Potts and Ganendra. It is also observed that the difference in accuracy for the two integrations is not significant. The sub-stepping algorithm shows slightly better accuracy for smaller increment sizes but faster deterioration for larger increment sizes.

\subsection{Stress-controlled cyclic triaxial tests}

In this section, the performance of the two integrations for stress-controlled cyclic behaviours is investigated. Values of the model constants are those that are appropriate for prediction of the Newfield clay, as listed in Table 3 . The specimen is hydrostatically consolidated with the confining pressure $p_{0}=400 \mathrm{kPa}$.

The stress-strain response and the stress path of the Newfield clay, which is subjected to one-way cyclic axial loading with a magnitude of $150 \mathrm{kPa}$ by using the implicit integration scheme, are presented in Fig. 7. It is found that the stress path almost becomes stable and the accumulation rate of the plastic strains decreases. This finding means that the cyclic shakedown is obtained at the cyclic stress level. The simulation results using the increment sizes of $\Delta q=1.5 \mathrm{kPa}$ and $\Delta q=0.75 \mathrm{kPa}$ are close each other. Again, the accuracy and stability of the return mapping algorithm are verified in predicting the cyclic behaviour of the saturated clay. Similar analysis is also conducted by using the substepping algorithm.

To investigate the efficiency of the two integration algorithms, more simulation of the stress-controlled cyclic loading at different cyclic levels with various stress increments is performed. The CPU 


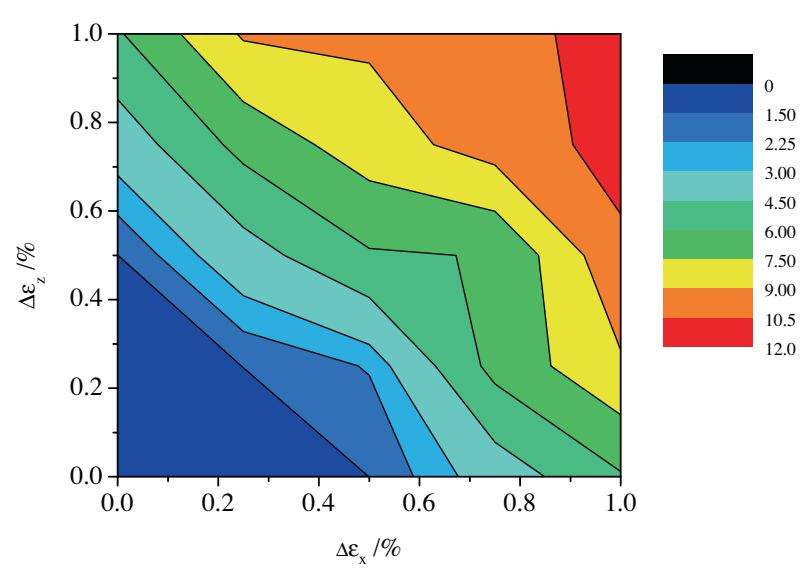

Fig. 5. Iso-error map from the return mapping integration scheme.

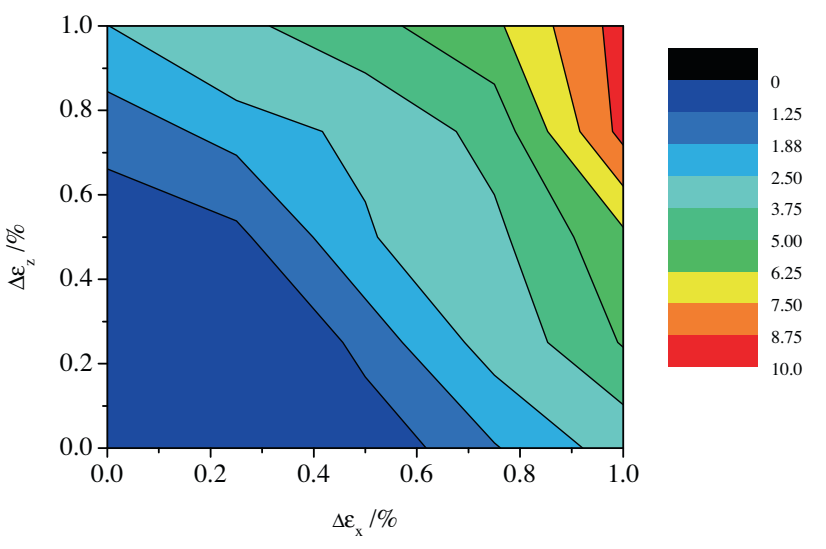

Fig. 6. Iso-error map from the sub-stepping integration scheme.

time required for each analysis is summarised in Table 5, in which NC means "not convergent". It is observed that predicting the cyclic behaviour of saturated clay by using the sub-stepping integration requires less CPU time than by using the return mapping integration. At a relatively large stress increment size, the sub-stepping integration cannot make itself convergent. These conclusions are consistent with the study by Manzari and Prachathananukit [18], in which a two-surface model for predicting the monotonic behaviour of sands was adopted.

\subsection{Strain-controlled cyclic triaxial tests}

The finite element model described in Section 5.3 is also used to perform a strain-controlled cyclic loading at a level of $\varepsilon_{1 d}= \pm 1.0 \%$. To demonstrate the application of the model to the boundary value problems, the finite element model is meshed into 8 elements. Values of the model constants are those that are appropriate for prediction of the marine plastic clay, as listed in Table 3. The specimen is hydrostatically consolidated with the confining pressure $p_{0}=210 \mathrm{kPa}$.

The simulation of the marine plastic clay using the sub-stepping scheme at different increment sizes is presented in Figs. 8 and 9. It is observed from Fig. 8 that the stress-strain relations move toward the strain axis, and the stiffness degradation occurs from the second load cycle. Comparing the results at different increment sizes, the accuracy of the sub-stepping integration scheme can be further confirmed.

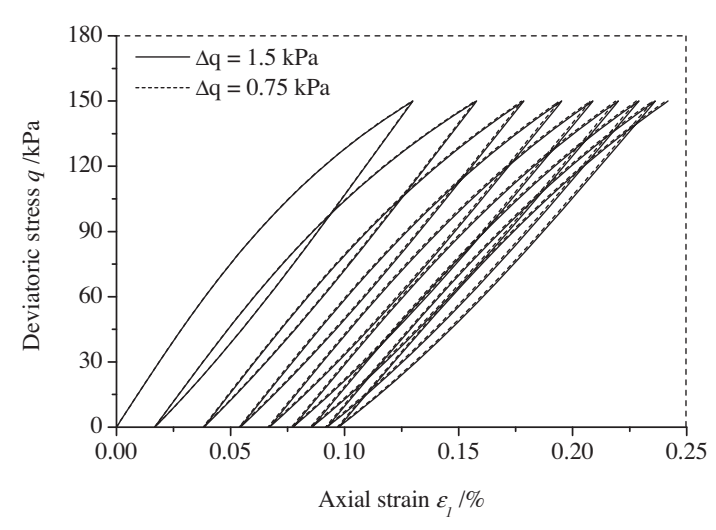

(a) Stress-strain relations

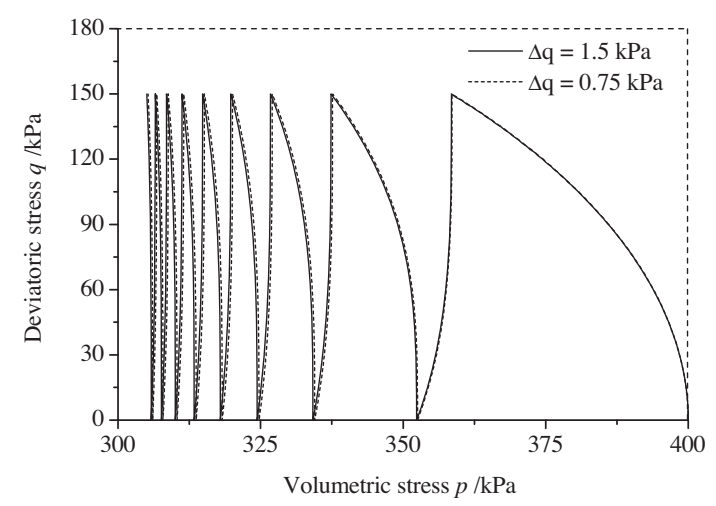

(b) Effective stress path

Fig. 7. Simulations of stress-controlled cyclic triaxial tests using the return mapping integration scheme.

Table 5

Comparison of the efficiency of the two integration schemes in stress-controlled cyclic triaxial tests.

\begin{tabular}{lrrll}
\hline Case & $q_{d}(\mathrm{kPa})$ & Increments & $\begin{array}{l}\text { Return mapping } \\
\text { CPU time }(\mathrm{s})\end{array}$ & $\begin{array}{l}\text { Sub-stepping } \\
\text { CPU time }(\mathrm{s})\end{array}$ \\
\hline 1 & 70 & 17,000 & 1167.4 & 1077.6 \\
2 & 70 & 1700 & 65.9 & 59.1 \\
3 & 100 & 17,000 & 1116.1 & 1058.3 \\
4 & 100 & 1700 & 63.4 & 60.4 \\
5 & 150 & 3400 & 131.2 & 122.5 \\
6 & 150 & 1700 & 62.4 & $\mathrm{NC}$ \\
7 & 200 & 3400 & 131.2 & 121.6 \\
8 & 200 & 1700 & 61.7 & $\mathrm{NC}$ \\
\hline
\end{tabular}

Similar to the simulation of the stress-controlled cyclic loading, another set of strain-controlled cyclic triaxial tests using the two integration schemes is conducted to further investigate the efficiency. To make the sub-stepping scheme convergent, the strain increments adopted here are relatively small. The CPU time required for each analysis is listed in Table 6. Similarly, it is observed that predicting the cyclic behaviour of soils that use the sub-stepping integration requires less CPU time than using the return mapping scheme.

\subsection{FEM analysis of a square footing under monotonic loading}

The collapse of a rigid footing is a well-known problem for testing stress integration. To demonstrate the application of the model with the two integration schemes to the bounding value problems, a finite element coupled analysis of a rigid square footing on the 


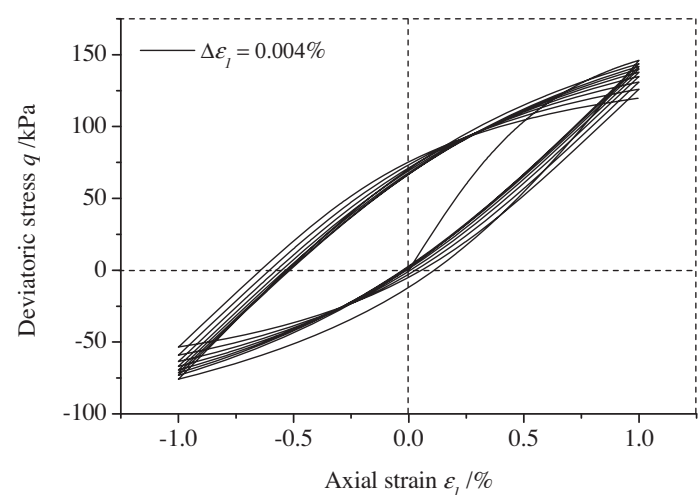

(a)

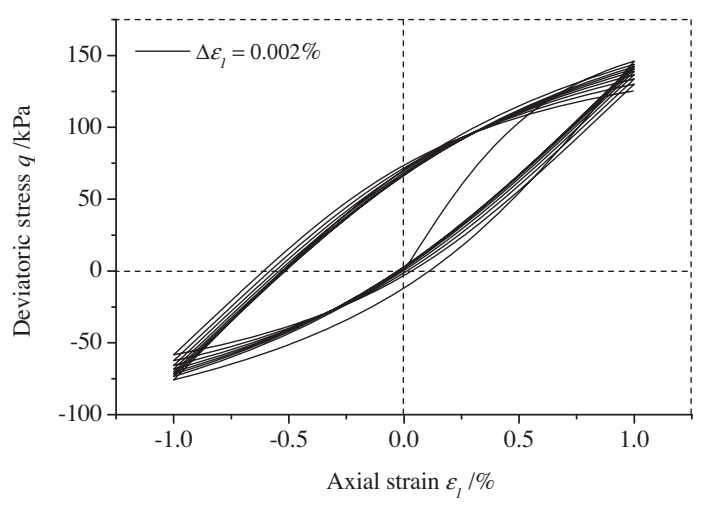

(b)

Fig. 8. The predicted stress-strain relations for strain-controlled cyclic triaxial tests using the sub-stepping algorithm.

Kaolin clay is performed. As illustrated in Fig. 10, the finite element model is composed of 7220 elements and 8400 nodes. Dimensions of the soil and the footing are $10 \mathrm{~m} \times 10 \mathrm{~m} \times 10 \mathrm{~m}$ and $0.68 \mathrm{~m} \times$ $0.68 \mathrm{~m}$, respectively. The lateral boundaries of the soil are fixed in both the $\mathrm{X}$ and $\mathrm{Y}$ directions but are allowed to move in the $\mathrm{Z}$ direction, and the bottom boundary is locked in all directions. The model constants of the soil are listed in Table 3. One additional parameter in the coupled analysis is the permeability $k_{p}$. In the present work, its value is set to $10^{-9} \mathrm{~m} / \mathrm{s}$, which is a typical value suggested by Potts and Zdravkovic [32] in the coupled consolidation finite element analysis. Considering that the footing is regarded as a rigid body, the displacement increments are actually applied at the soil boundaries that are in contact with the footing. To avoid any interface elements in the 3D finite element analysis, the soil-footing interface is treated as completely smooth with free horizontal displacements at the contact nodes.

Note that most of the available analyses on the bearing capacity of the square footing are for the clay with a uniform strength and under undrained conditions [33-36]. To compare them, two special steps in the present analysis are necessary. First, in the geostatic step, the soil is hydrostatically consolidated under a pressure of $110 \mathrm{kPa}$ to obtain clay with a uniform strength. In the bounding surface plasticity model, which is based on critical state theory, for a given group of critical state parameters $(\kappa, \lambda, M)$, the initial undrained strength of saturated clay after consolidation is determined by the initial size of the bounding surface (i.e., the pre-consolidation pressure $p_{c}$ ). Second, in the coupled analysis step, fast loading is necessary to retain an undrained condition. Here, the loading rate defined by Sheng et al. [37] is adopted

$\omega=\frac{\Delta w / t}{k_{p} \gamma_{w}}$

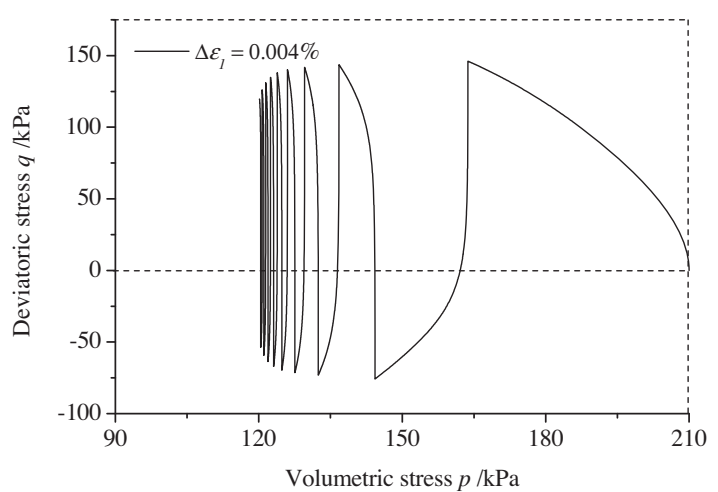

(a)

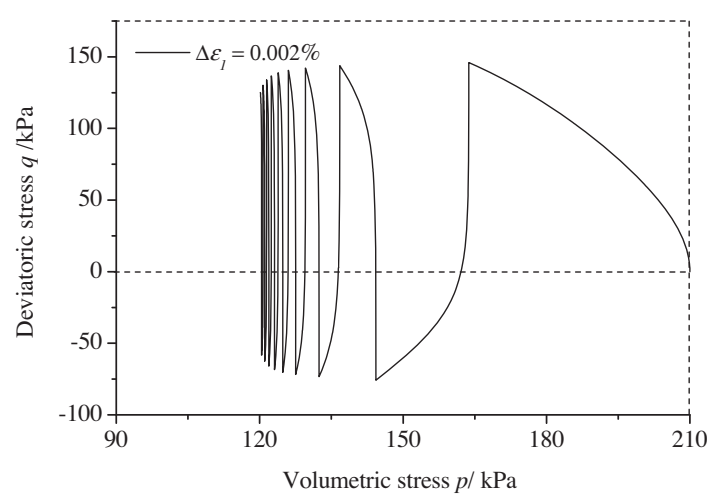

(b)

Fig. 9. The predicted stress path for strain-controlled cyclic triaxial tests using the sub-stepping algorithm.

Table 6

Comparison of the efficiency of the two integration schemes in strain-controlled cyclic triaxial tests.

\begin{tabular}{llrll}
\hline Case & $\varepsilon_{1 d}(\%)$ & Increments & $\begin{array}{l}\text { Return mapping } \\
\text { CPU time }(\mathrm{s})\end{array}$ & $\begin{array}{l}\text { Sub-stepping } \\
\text { CPU time }(\mathrm{s})\end{array}$ \\
\hline 1 & 0.3 & 6400 & 323.5 & 305.5 \\
2 & 0.3 & 3200 & 156.3 & 130.4 \\
3 & 0.5 & 6400 & 357.4 & 319.2 \\
4 & 0.5 & 3200 & 150.5 & 131.9 \\
5 & 1.0 & 16,500 & 1038 & 968.7 \\
6 & 1.0 & 8250 & 409.1 & 342.7 \\
7 & 1.5 & 6400 & 362 & 332.1 \\
8 & 1.5 & 3200 & 164.6 & 131.4 \\
\hline
\end{tabular}

where $\Delta w$ is the equivalent footing pressure applied over the time period $t$; and $\gamma_{w}$ is the unit weight of water. Sheng et al. [37] considered that the soil behaves essentially in an undrained manner when the loading rate $\omega$ equals $10^{4}$. Thus, in the present work, the loading rate $\omega$ is set to $10^{5}$. In ABAQUS, the option of automatic time incrementing is open because the fixed increments could prevent the solution from converging. As a result, the numbers of increments in the explicit and implicit integration schemes are usually different. In a coupled analysis, the pore pressure tolerance (i.e., UTOL in ABAQUS), which specifies the allowable pore pressure change per increment, has a greater influence on the global convergence than the displacement tolerance. In the present work, by trial and error, tolerances in the pore pressure and in the displacement are set to $5 \mathrm{kPa}$ and $10^{-5}$, respectively.

The normalised load-displacement curves by using the two integration schemes are presented in Fig. 11. It can be observed that each of the two algorithms produces a reasonable analysis 


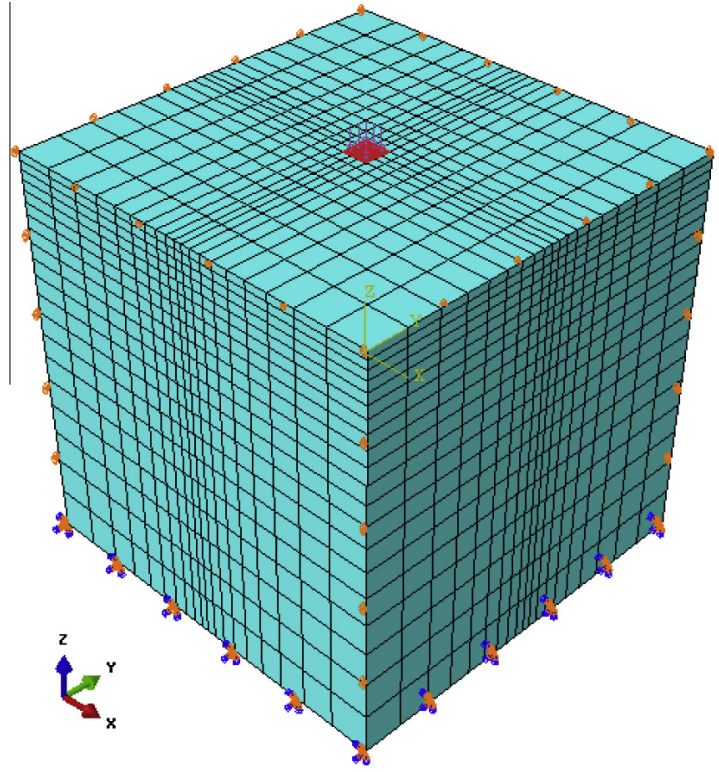

Fig. 10. Finite element model used in simulating the square footing under monotonic loading.

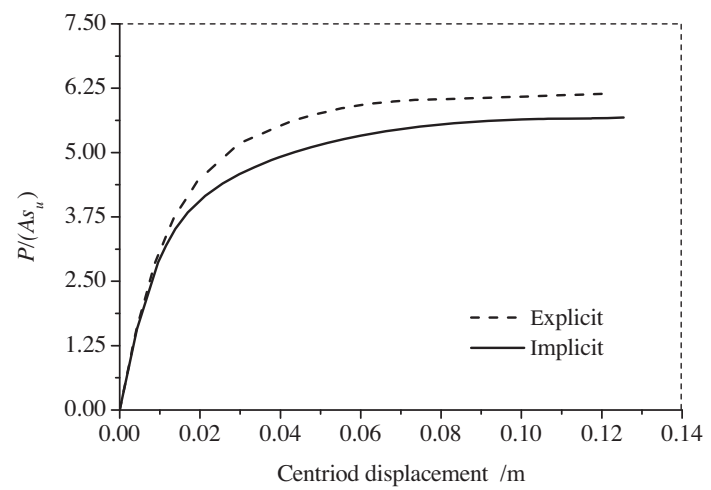

Fig. 11. Normalized load-displacement response of the rigid square footing on normally consolidated Kaolin clay.

of the footing problem. At the same time, the departure between them is higher than that at the element level (see Fig. 4). This finding could be attributed to the difference in the number of increments in the two integrations. Note that the bearing capacity factor $N=P /\left(A s_{u}\right)$ can be determined from the curve at the point where it becomes almost linear [38]. Then, the values of $N$ from the Skempton's expression, other available analytical and numerical solutions for rigid square footings and the present integration schemes are compared in Table 7. The bearing capacity of the footing predicted by the sub-stepping algorithm is slightly larger than
Table 8

Comparison of the efficiency of the two integration schemes for the square footing under monotonic loading.

\begin{tabular}{lll}
\hline Algorithms & Global iterations & CPU time $(\mathrm{s})$ \\
\hline Return mapping & 1489 & 16581 \\
Sub-stepping & 1673 & 14335 \\
\hline
\end{tabular}

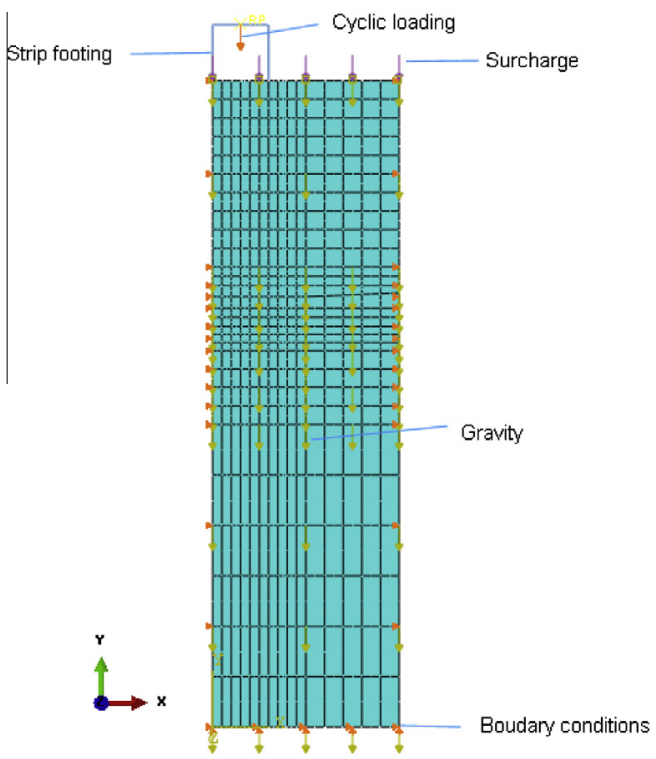

Fig. 12. Finite element model used in simulating the strip footing under cyclic loading.

the other results except for Skempton's expression. The CPU usage of the analysis by using the two integrations is listed in Table 8 . Again, it is found that the sub-stepping integration is more efficient than the implicit integration at the specified increment size.

\subsection{FEM analysis of a strip footing under long-term cyclic loading}

A cyclic test on a strip footing [39] is simulated to further demonstrate the capability of the model in solving the boundary value problems that involve long-term cyclic loading. In the test, a strip foundation with the size of $7.61 \mathrm{~cm} \times 22.9 \mathrm{~cm} \times 3.81 \mathrm{~cm}$ was supported by saturated clay and subjected to a static load of $17.4 \mathrm{kPa}$, which superimposed a cyclic load of $2.54 \mathrm{kPa}$. The model parameters are listed in Table 3. Details of the cyclic test can be found in the literature [39].

Plane strain conditions are assumed, and a symmetrical 2D finite element model is established, as illustrated in Fig. 12. Note that the soil is under its own weight and an additional surcharge of $19 \mathrm{kPa}$, through which the strength of the clay varies with depth.

Table 7

Comparison of the bearing capacity factor of the square footing.

\begin{tabular}{|c|c|c|c|}
\hline Reference & Analysis type & & $N$ \\
\hline Skempton [32] & Empirical & & 6.17 \\
\hline Shield and Drucker [33] & Upper bound & & 5.71 \\
\hline Michalowski and Dawson [34] & Finite difference & & 5.43 \\
\hline Gourvenec et al. [35] & Finite element (Tresca soil model) & & 5.56 \\
\hline \multirow[t]{2}{*}{ Present study } & Finite element (BSP ${ }^{\mathrm{a}}$ model $)$ & Return mapping & 5.61 \\
\hline & & Sub-stepping & 6.02 \\
\hline
\end{tabular}

\footnotetext{
a Bounding surface plasticity.
} 


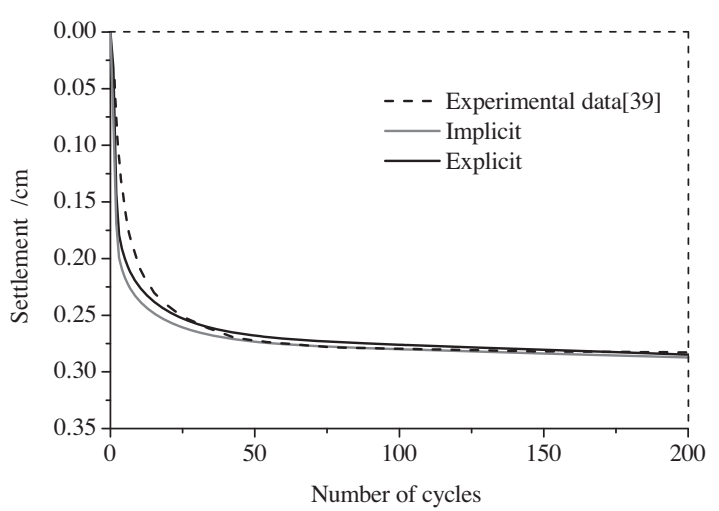

Fig. 13. The settlement accumulation of the strip footing under cyclic loading.

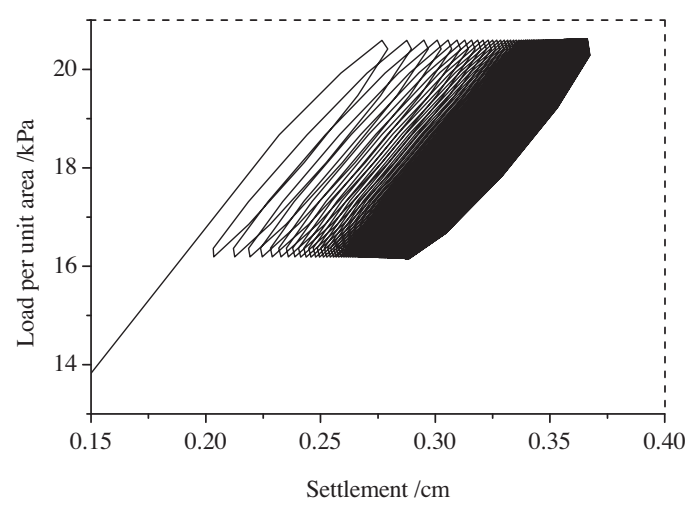

Fig. 14. The cyclic load-settlement curve calculated by the implicit integration.

Table 9

Comparison of the efficiency of the two integration schemes for the strip footing under cyclic loading.

\begin{tabular}{ll}
\hline Algorithms & CPU time (s) \\
\hline Return mapping & 1219.5 \\
Sub-stepping & 1163.2 \\
\hline
\end{tabular}

The footing is regarded as a rigid body and is tied to the soil elements.

Fig. 13 shows the settlement accumulation with the number of loading cycles. It is observed that the simulation results from both the implicit and explicit integrations are generally coincident with the experimental data. At the initial loading stage, the displacement accumulates rapidly. Then, the rate of accumulation decreases with the number of cycles until it is almost zero, which indicates that the cyclic shakedown has been approached. The cyclic load-settlement curve from the implicit scheme is demonstrated in Fig. 14, which shows that the hysteresis loop is approximately closed at the state of cyclic shakedown. The CPU usage by the two integrations is listed in Table 9. In the simulation, a fixed load increment is adopted and a repeated attempt is made to make both of the integrations convergent. It can be seen from Table 9 that for the cyclic bearing behaviour of the strip footing, the implicit return mapping integration is more time-consuming than the sub-stepping integration.

\section{Conclusions}

In the present work, two stress point integration algorithms, i.e., the implicit return mapping and the explicit sub-stepping schemes, are adopted in the anisotropic bounding surface plasticity model with a vanishing elastic region, and the performance is evaluated both at the soil element level and in solving boundary value problems. For this reason, there is no explicit current yield surface in the bounding surface model to which the conventional implicit algorithm returns the stress state back or to which the sub-stepping integration corrects the drift of the stress state; several modifications have been made for both of these integration schemes. First, the image stress point is mapped or corrected to the bounding surface instead of mapping back or correcting the stress state to the yield surface. Second, the unloading-loading criterion is checked to determine the image stress point rather than checking the yield criterion after giving the trial stress state in a conventional way.

To assess the performance of the integration algorithms in the present model, a series of numerical simulations of conventional triaxial tests, stress-controlled and strain-controlled cyclic triaxial tests as well as boundary value problems that involve monotonic and cyclic bearing behaviours of rigid footings on normally consolidated saturated clay were conducted. The results indicate that there is no significant difference in the accuracy between the implicit and explicit integrations for smaller strain increments, but the explicit integration shows a higher efficiency. For relatively larger increment sizes, the implicit return mapping algorithm shows better accuracy and convergence, while the sub-stepping suffers the convergence problem that is attributed to the continuum tangent matrix being adopted. Furthermore, with the tolerance used in the present model, the soil strength and the bearing behaviour of the footing predicted by the sub-stepping algorithm are slightly larger than those by the return mapping algorithm. The model is a representative of a series of bounding surface models that have typical characteristics, including the isotropic and kinematic hardening, pressure dependency of the elastic bulk and shear moduli, and a rotational bounding surface to capture complex but important cyclic behaviours of soils, such as the cyclic shakedown and degradation. The present work could provide a guide for similar attempts in this class of bounding surface plasticity models.

\section{Acknowledgments}

Financial support from the National Natural Science Foundation of China (Grant nos. 50979070 and 51179124) is gratefully acknowledged.

\section{Appendix A. Consistency condition on the current stress point}

As shown in Fig. A1, passing the current stress point $\boldsymbol{\sigma}$ and the homological centre (i.e., the mapping origin o), there is a dependent surface $f_{m}$ implied in the model, which is homologous to the bounding surface $F_{m}$.

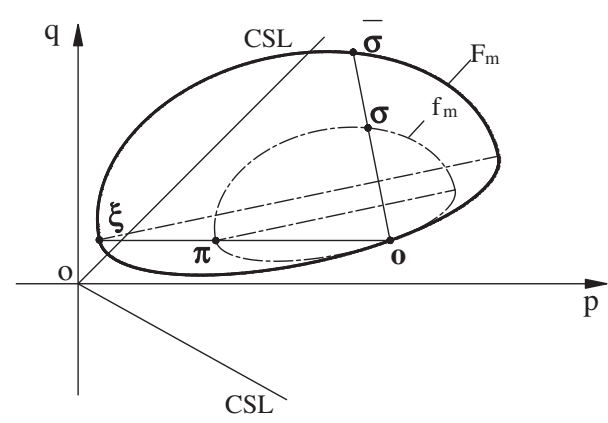

Fig. A1. The condition of consistency on the surface $f_{m}$ implied in the model. 
From the mapping rule and geometric similarity between the two surfaces, we have:

$$
\left\{\begin{array} { l } 
{ \overline { p } = o _ { p } + b ( p - o _ { p } ) } \\
{ \overline { \mathbf { s } } = \mathbf { o } _ { s } + b ( \mathbf { s } - \mathbf { o } _ { s } ) }
\end{array} \text { and } \left\{\begin{array}{l}
\xi_{p}=o_{p}+b\left(\pi_{p}-o_{p}\right) \\
\xi_{s}=\mathbf{o}_{s}+b\left(\pi_{s}-\mathbf{o}_{s}\right)
\end{array}\right.\right.
$$

where $\left(\pi_{p}, \pi_{s}\right)$ denotes the corresponding endpoint of $f_{m}$.

Substituting Eq. (A1) into the formation of the bounding surface (i.e., Eq. (3)), obtain with

$$
\begin{aligned}
& e_{1}=2 C_{4}-p_{c}-3 C C_{6}, \mathbf{e}_{2}=3 C b \overline{\mathbf{s}}, \\
& e_{3}=-C_{4}, e_{4}=\left(2 C_{4}-p_{c}-3 C C_{6}\right) C_{5}+3 C C_{10}
\end{aligned}
$$

From Eq. (14i), we obtain

$f_{1} \Delta p+\mathbf{f}_{2}: \Delta \mathbf{s}+f_{3} \Delta p_{c}+f_{4} \Delta b+f_{6} \Delta \bar{K}_{p}=0$

with

$$
\left\{\begin{array}{l}
f_{1}=Z_{0} p_{c} \bar{n}_{p} b+2 M^{2} C C_{4} b p_{c} \chi_{0}+3 C C_{4} C_{6} b p_{c} \frac{\beta}{\hat{q}}, \mathbf{f}_{2}=-\left(3 C C_{4} b p_{c} \chi_{0} \boldsymbol{\alpha}+3 C C_{4} b p_{c} \frac{\beta}{\hat{q}} \hat{\mathbf{s}}\right) \\
f_{3}=\left(Z_{0} C_{4} \bar{n}_{p}-C_{4} p_{c} \chi_{0}\right), f_{4}=Z_{0} p_{c} \bar{n}_{p} C_{5}+C C_{4} p_{c} \chi_{0}\left(2 M^{2} C_{5}-3 C_{1}\right)+3 C C_{4} C_{9} p_{c} \frac{\beta}{\tilde{q}}, f_{6}=-1
\end{array}\right.
$$

$$
\left\{\begin{array}{l}
F_{m}=b^{2} f_{m}=0 \\
f_{m}=\left(p-\pi_{p}\right)^{2}-\left(p-\pi_{p}\right) \frac{p_{c}}{b}+\frac{3}{2\left(M^{2}-\alpha^{2}\right)}\left[\mathbf{s}-\pi_{s}-\left(p-\pi_{p}\right) \boldsymbol{\alpha}\right]:\left[\mathbf{s}-\pi_{s}-\left(p-\pi_{p}\right) \boldsymbol{\alpha}\right]=0
\end{array}\right.
$$

By taking the time-derivative of $F_{m}$, the following expression is derived:

$\dot{F}_{m}=b^{2} \dot{f}_{m}+2 b f_{m} \dot{b}=0$

Because $f_{m}=0$, it follows that $\dot{f}_{m}=0$. This result means that the condition of consistency on $F_{m}$ ensures the condition of consistency on $f_{m}$, which the current stress state lies on. It should be clarified that $f_{m}$ is actually implied in the model, and its evolution is totally determined by the bounding surface and the current stress state.

\section{Appendix B. Consistent tangent operator}

From Eq. (14a), we obtain

$a_{1} \Delta p+\mathbf{a}_{2}: \Delta \mathbf{s}+a_{3} \Delta p_{c}+a_{4} \Delta b+a_{5} \Delta \Lambda=\Delta \varepsilon_{v}$

with

$$
\begin{aligned}
a_{1} & =2 C M^{2} \Lambda b+\frac{1}{K}, \mathbf{a}_{2}=-3 C \mathbf{\alpha} \Lambda b, a_{3}=-\Lambda, a_{4} \\
& =\Lambda C\left(2 M^{2} C_{5}-3 C_{1}\right), a_{5}=\bar{n}_{p}
\end{aligned}
$$

From Eq. (14c), we obtain

$\mathbf{b}_{1} \Delta p+b_{2} \Delta \mathbf{s}+\mathbf{b}_{4} \Delta b+\mathbf{b}_{5} \Delta \Lambda=\Delta \mathbf{e}$

with

$\mathbf{b}_{1}=-3 C \boldsymbol{\alpha} \Lambda b, b_{2}=3 C \Lambda b+\frac{1}{2 G}, \mathbf{b}_{4}=3 C \Lambda \widetilde{\mathbf{s}}, \mathbf{b}_{5}=\overline{\mathbf{n}}_{s}$

From Eq. (14e), we obtain

$c_{1} \Delta p+\mathbf{c}_{2}: \Delta \mathbf{s}+c_{3} \Delta p_{c}=p_{c} \chi_{0} \Delta \varepsilon_{v}-\frac{2}{3} \beta p_{c} \frac{\Delta \mathbf{e}^{p}}{\Delta \varepsilon_{A}} \Delta \mathbf{e}$

with

$c_{1}=\frac{p_{c} \chi_{0}}{K}, \mathbf{c}_{2}=-\frac{\beta p_{c}}{3 G} \frac{\Delta \mathbf{e}^{p}}{\Delta \varepsilon_{A}}, c_{3}=1$

From Eq. (14g), we obtain

$d_{1} \Delta p+\mathbf{d}_{2}: \Delta \mathbf{s}+d_{3} \Delta p_{c}+d_{4} \Delta b+d_{5} \Delta \Lambda+d_{6} \Delta K_{p}=0$

with

$$
\left\{\begin{array}{l}
d_{1}=\frac{\Lambda C_{2}}{A}\left(2 C_{7}+p_{c}-3 C C_{8}\right), \mathbf{d}_{2}=3 C \frac{\Lambda C_{2}}{A} \tilde{\xi}, d_{3}=\frac{\Lambda C_{2}}{A} C_{5} \\
d_{4}=\frac{\Lambda}{A} K_{p}+1+\frac{\Lambda}{A} \gamma b \rho(b-1)^{(\gamma-1)}, d_{5}=-C_{2}, d_{6}=-C_{3}
\end{array}\right.
$$

From Eq. (14h), we obtain

$e_{1} \Delta p+\mathbf{e}_{2}: \Delta \mathbf{s}+e_{3} \Delta p_{c}+e_{4} \Delta b=0$
In the above equations, we define

$$
\left\{\begin{array}{l}
C=\frac{1}{M^{2}-\alpha^{2}}, C_{1}=\left(\overline{\mathbf{s}}-\mathbf{o}_{s}\right) \boldsymbol{\alpha}, C_{2}=\frac{\bar{K}_{p}-b K_{p}}{A}, C_{3}=\frac{\Lambda}{A}\left[1-b+b(b-1)^{r}\right] \\
C_{4}=\bar{p}-\xi_{p}, C_{5}=p-o_{p}, C_{6}=\hat{\mathbf{s}}: \boldsymbol{\alpha}, C_{7}=\xi_{p}-o_{p}, C_{8}=\tilde{\xi}: \boldsymbol{\alpha}, C_{9}=\hat{\mathbf{s}}: \tilde{\mathbf{s}} \\
C_{10}=\hat{\mathbf{s}}:\left(\overline{\mathbf{s}}-\mathbf{o}_{s}\right), \tilde{\xi}=\left(\xi_{s}-\mathbf{o}_{s}\right)-\left(\xi_{p}-o_{p}\right) \boldsymbol{\alpha}, \tilde{\mathbf{s}}=\left(\mathbf{s}-\mathbf{o}_{s}\right)-\left(p-o_{p}\right) \boldsymbol{\alpha} \\
Z_{0}=\chi_{0}-2 \beta C \frac{\bar{q}}{\bar{n}_{p}}, \bar{q}=\sqrt{\frac{3}{2}} \hat{\mathbf{s}}: \hat{\mathbf{s}}
\end{array}\right.
$$

From Eqs. B1, B3, B5, B7, and B9, we have

$$
\left[\begin{array}{cccccc}
a_{1} & \mathbf{a}_{2}^{T} & a_{3} & a_{4} & a_{5} & 0 \\
\mathbf{b}_{1} & b_{2} \mathbf{I}^{T} & \mathbf{0} & \mathbf{b}_{4} & \mathbf{b}_{5} & \mathbf{0} \\
c_{1} & \mathbf{c}_{2}^{T} & c_{3} & 0 & 0 & 0 \\
d_{1} & \mathbf{d}_{2}^{T} & d_{3} & d_{4} & d_{5} & d_{6} \\
e_{1} & \mathbf{e}_{2}^{T} & e_{3} & e_{4} & 0 & 0 \\
f_{1} & \mathbf{f}_{2}^{T} & f_{3} & f_{4} & 0 & f_{6}
\end{array}\right]\left[\begin{array}{c}
\Delta p \\
\Delta \mathbf{s} \\
\Delta p_{c} \\
\Delta b \\
\Delta \Lambda \\
\Delta \bar{K}_{p}
\end{array}\right]=\left[\begin{array}{c}
\Delta \varepsilon_{v} \\
\Delta \mathbf{e} \\
p_{c} \chi_{0} \Delta \varepsilon_{v}-\frac{2 \beta p_{c}}{3 \Delta \varepsilon_{A}} \Delta \mathbf{e}^{p}: \Delta \mathbf{e} \\
0 \\
0 \\
0
\end{array}\right]
$$

Therefore, the consistent tangent modulus $\mathbf{C}_{n+1}^{k}$ can be obtained by solving $\frac{\partial \Delta p_{n+1}^{k}}{\partial \Delta \varepsilon_{n+1}^{k}}$ and $\frac{\partial \Delta \mathbf{s}_{n+1}^{k}}{\partial \Delta \varepsilon_{n+1}^{k}}$.

\section{References}

[1] Singh SP, Ramaswamy SV. Response of plate anchors to sustained-cyclic loading. Indian Geotech J 2002;32(2):161-72.

[2] Chen W, Randolph MF. Uplift capacity of suction caissons under sustained and cyclic loading in soft clay. J Geotech Geoenviron Eng 2007;133(11):1352-63.

[3] Anderson KH. Bearing capacity under cyclic loading-offshore, along the coast and on land. Can Geotech J 2009;46:513-35.

[4] Simo JC, Taylor RL. Consistent tangent operators for rate-independent elastoplasticity. Comput Methods Appl Mech Eng 1985;48:101-18.

[5] Simo JC, Taylor RL. Return mapping algorithm for plane stress elasto-plasticity. Int J Numer Methods Eng 1986;22:649-70.

[6] Simo JC, Hughes T. Computational inelasticity: interdisciplinary applied mathematics series. Springer; 2000.

[7] Manzari TM, Noor MA. On implicit integration of bounding surface plasticity models. Comput Struct 1997;63:385-95.

[8] Rouainia M, Wood DM. Implicit numerical integration for a kinematic hardening soil plasticity model. Int J Numer Anal Meth Geomech 2001;25: $1305-25$.

[9] Borja RI, Lin CH, Montans FJ. Cam-clay plasticity. Part IV: implicit integration of anisotropic bounding surface model with nonlinear hyper-elasticity and ellipsoidal loading function. Comput Methods Appl Mech Eng 2001;190: 3293-323.

[10] Tamagnini C, Castellanza R, Nova R. A generalized backward Euler algorithm for the numerical integration of an isotropic hardening elastoplastic model for mechanical and chemical degradation of bonded geo-materials. Int J Numer Anal Meth Geomech 2002;26:963-1004.

[11] Sloan SW. Substepping scheme for numerical integration of elastoplastic stress-strain relations. Int J Numer Methods Eng 1987;24:893-911.

[12] Sloan SW, Abbo AJ, Sheng DC. Refined explicit integration of elastoplastic models with automatic error control. Eng Comput 2001;18(1/2):121-54.

[13] Abbo AJ, Sloan SW. An automatic load stepping algorithm with error control. Int J Numer Methods Eng 1996;39:1737-59. 
[14] Sheng DC, Sloan SW. Loading stepping schemes for critical state models. Int J Numer Methods Eng 2001;50:67-93.

[15] Zhao J, Sheng D, Rouainia M, Sloan SW. Explicit stress integration of complex soil models. Int J Numer Anal Meth Geomech 2005;29:1209-29.

[16] Andrianopoulos KI, Papadimitriou AG, Bouckovalas GD. Explicit integration of bounding surface model for the analysis of earthquake soil liquefaction. Int J Numer Anal Meth Geomech 2009;34(15):1586-614.

[17] Potts D, Ganendra D. An evaluation of substepping and implicit stress point algorithms. Comput Methods Appl Mech Eng 1994;119:341-54.

[18] Manzari MT, Prachathananukit R. On integration of cyclic soil plasticity model. Int J Numer Anal Meth Geomech 2001;25(6):525-49.

[19] Sołowski WT, Hofmann M, Hofstetter G, Sheng DC, Sloan SW. A comparative study of stress integration methods for the Barcelona Basic Model. Comput Geotech 2012;44:22-33.

[20] Li T, Meissner H. Two-surface plasticity model for cyclic undrained behavior of clays. J Geotech Geoenviron Eng 2002;128(7):613-26.

[21] Mroz Z, Norris VA, Zienkiewicz OC. An anisotropic hardening model for soils and its application to cyclic loading. Int J Numer Anal Meth Geomech 1978;2(3):203-21.

[22] Elgamal A, Yang Z, Parra E. Computational modeling of cyclic mobility and post-liquefaction site response. Soil Dynam Earthq Eng 2002;22(4):259-71.

[23] Hu C, Liu HX, Huang W. Anisotropic bounding-surface plasticity model for the cyclic shakedown and degradation of saturated clay. Comput Geotech 2012;44:34-47.

[24] Dafalias YF. An anisotropic critical state clay plasticity model. Mech Res Commun 1986;13:341-7.

[25] Anandarajah A, Dafalias YF. Bounding surface plasticity. III: Application to anisotropic cohensive soils. J Eng Mech, ASCE 1986;112(12):1292-318.

[26] Liang RY, Ma F. Anisotropic plasticity model for undrained cyclic behavior of clays. II: Verification. J Geotech Eng 1992;118(2):246-65.
[27] Ling HI, Yue D, Kaliakin VN, Themelis NJ. Anisotropic elasto-plastic bounding surface model for cohesive soils. J Eng Mech, ASCE 2002;129(7): 748-58.

[28] Huang MS, Liu YH, Sheng DC. Simulation of yielding and stress-strain behavior of shanghai soft clay. Comput Geotech 2011;38:341-53.

[29] Oh S, Lee SR. Formulation of implicit stress integration and consistent tangent modulus for an anisotropic hardenging constitutive model. Comput Methods Appl Mech Eng 2001;191:255-72.

[30] Hibbit, Karlsson, Sorensen, Inc (HKS). ABAQUS users' manual, version 6.7.1; 2009.

[31] Stipho ASA. Experimental and theoretical investigation of the behavior of ansiotropically consolidated kaolin. PhD Thesis, Univ College Cardiff, UK; 1978.

[32] Potts DM, Zdravkovic L. Finite element analysis in geotechnical engineering: theory \& application. Thomas Telford Publisher; 2001.

[33] Skempton AW. The bearing capacity of clays. In: Proc. building and research congress, vol. 1, London; 1951. p. 180-9.

[34] Shield RT, Drucker DC. The application of limit analysis to punch indentation problems. J Appl Mech 1953;20:453-60.

[35] Michalowski RL, Dawson EM. Three-dimensional analysis of limit loads on Mohr-Coulomb soil, vol. 1. Poland: Foundations of Civil and Environmental Engineering, Poznan University of Technology Press; 2002. p. 137-47.

[36] Gourvenec S, Randolph MF, Kingsnorth O. Undrained bearing capacity of square and rectangular footings. Int J Geomech, ASCE 2006;6(3): 147-57.

[37] Sheng DC, Sloan SW, Yu HS. Aspects of finite elment implementation of critical state models. Cmput Mech 2000;26:185-96.

[38] Vesic AS. Analysis of ultimate loads on shallow foundations. J Soil Mech Found Division, ASCE 1973;99:45-73.

[39] Das BM, Shin EC. Laboratory model tests for cyclic load-induced settlement of a strip foundation on a clayey soil. Geotech Geol Eng 1996;4:213-25. 